\title{
Radiation transport within oceanic (case 1) water
}

\author{
André Morel and Bernard Gentili \\ Laboratoire d'Océanographie de Villefranche, Université Pierre et Marie Curie/CNRS, Villefranche-sur-mer, France
}

Received 19 December 2003; revised 10 March 2004; accepted 5 April 2004; published 4 June 2004.

[1] A spectral model of the inherent optical properties (IOP) of oceanic case 1 waters, as previously developed for studying the near-surface bidirectional reflectance, provides the input parameters for the present computations of radiative transport (RT), now extended throughout the water column (three times the euphotic zone). All spectral apparent optical properties (AOP) are computed at each of the levels (30) for six chlorophyll values (from 0.03 to $10 \mathrm{mg} \mathrm{m}^{-3}$ ) and for six values of the zenith Sun angle (from $0^{\circ}$ to $75^{\circ}$ ). The Raman emission is accounted for. From the irradiances and radiances values the various attenuation coefficients $(K)$, the average cosines $(\bar{\mu})$, and the reflectance $(R)$ are derived for all depths and layers. Their variations resulting from the Sun's position are also studied, which removes the static character of previous empirical models inasmuch as the diurnal changes of the parameters describing the in-water light field can be predicted. The AOPs observed within the deepest levels are also compared to values independently derived from an asymptotic (iterative) solution of the RT. The rate of approach to the asymptotic regime is numerically analyzed; this rate is actually governed by $\tau_{b}$, i.e., this fraction of $\tau$ (the optical thickness) that corresponds only to scattering. Practical applications of these systematic computations are examined, such as the change (with solar position) of the euphotic depth, the time- and wavelength-dependent scalar irradiance that controls during the day the energy available for photosynthesis (or the heating rate), and the interpretation of radiometric field experiments involving upward and downward irradiance measurements. Some approximate expressions relating AOPs to IOPs are examined in the light of exact computations. INDEX TERMS: 4552 Oceanography: Physical: Ocean optics; 4203 Oceanography: General: Analytical modeling; 4847 Oceanography: Biological and Chemical: Optics; 4853 Oceanography: Biological and Chemical: Photosynthesis

Citation: Morel, A., and B. Gentili (2004), Radiation transport within oceanic (case 1) water, J. Geophys. Res., 109, C06008, doi:10.1029/2003JC002259.

\section{Introduction}

[2] Bio-optical models, such as those developed by Baker and Smith [1982], Gordon et al. [1988], and Morel [1988], have historically provided the first tools in the attempts at relating the optical properties of case 1 waters [Morel and Prieur, 1977] to the chlorophyll concentration (Chl). Recall that $\mathrm{Chl}$ is used as an indicator of the trophic state and more generally as an index of the whole biogenous material content (living or inanimate, pigmented or not) in case 1 waters. These models are "semiempirical" in nature [Gordon and Morel, 1983] to the extent that they rely on field data and specifically on empirical relationships statistically established between $\mathrm{Chl}$ and some bulk optical properties (apparent or inherent optical properties (AOPs and IOPs, respectively) (as defined by Preisendorfer [1961]).

[3] Concurrently, these models make use of several assumptions relative to missing or poorly documented parameters. Finally, they combine these inputs into a theoretical frame, itself based on numerical, often simplified, solutions of the radiative transfer equation

Copyright 2004 by the American Geophysical Union. 0148-0227/04/2003JC002259
(RTE). For this reason, such approaches can, as well, be coined as "semianalytical".

[4] Purely empirical models, essentially developed in view of deriving operational ocean color algorithms, have consisted of directly (i.e., statistically) relating Chl to the spectral reflectance without resorting to other optical quantities [see, e.g., Clarke and Ewing, 1974; Clark, 1981; O'Reilly et al., 1998; Bricaud et al., 2002; d'Ortenzio et al., 2002]. Standard processings (in terms of Chl) of the ocean color data, such as those provided by satellite-borne sensors (CZCS, SeaWiFs, or others), often rest on such straightforward methods, which are rather successful in case 1 waters but do not bring real understanding.

[5] In contrast, a purely analytical approach would ideally start with a thorough identification of all the optically significant components and their concentrations. Then, it would go on with the knowledge of the spectral IOPs (absorption, scattering, and volume scattering function) of each of these components; finally, it would require the operation of the RTE under prescribed boundary conditions, in view of deriving from the IOPs all the desired AOPs, and presenting them in a convenient form. Such models are presently impaired by an insufficient knowledge of the needed quantities (see discussion by Morel and Maritorena 
[2001]). In spite of stubborn attempts, this conceptually satisfying approach very likely will continue to come up against many obstacles before being fully successful (e.g., see the discussion by Stramski et al. [2001]). Analytical ways, however, are inescapable when dealing with the complex case 2 waters, even if several pieces of information are lacking so that various simplifications and assumptions must be accepted.

[6] The semianalytical approach was employed by Morel and Maritorena [2001] (hereinafter referred to as MM01) and mainly dealt with the diffuse attenuation coefficient and subsurface irradiance reflectance ( $K_{d}$ and $R$, respectively) (see symbols and definitions in Table 1) as a function of Chl. The present work, based on MM01, specifically aims at removing the "static" character of this previous model, namely its intrinsic insensitivity to external conditions. These conditions are essentially determined by the Sun position and the ratio of sky diffuse radiation to direct sunlight and, to a lesser extent, by the sea state. Indeed, the MM01 model, for it has been developed for mean lighting conditions, cannot directly answer questions such as, How much does the depth of the euphotic layer vary along the day? To what extent can the diffuse attenuation coefficients be considered as constant? How does the in-water light field change with depth and Sun position? Such questioning is put forward, for instance, by biologists interested in primary production or phytoplankton fluorescence as well by physicists interested in solar heating and mixed layer modeling.

[7] Actually, the influence of the Sun angle has already been studied in the case of some specific AOPs, namely the magnitude and the bidirectional structure of the upward radiance field just beneath (and just above) the ocean surface [Morel et al., 2002]. This first nonstatic study was prompted by the fact that the ocean reflectance anisotropy has direct implications in the ocean color remote sensing problem; therefore its results may have practical applications when processing ocean color data. For this study, mainly devoted to the upper oceanic layer from which the remotely sensed signal originates, the initial MM01 model was supplemented by considering the influence of Raman scattering and by making the shape of the particle phase function vary along with the chlorophyll concentration in case 1 waters.

[8] Nonetheless, besides reflectance, all other AOPs also depend in various ways on the prevailing illumination above the surface to an extent that must be quantified. Because of the progressive rearrangement of the radiant field within the water body, these AOPs are also varying as a function of the depth, even if their dependence with regard to the Sun position progressively weakens with increasing depth. The main purpose of the present study is to examine as function of $\mathrm{Chl}$ several important AOPs and their spectral behavior under dependence on the incident radiation geometry and to quantify the evolution of these AOPs along with depth, including their evolution toward the so-called asymptotic or diffusion regime [Preisendorfer, 1959; Gordon et al., 1993, and references therein].

\section{Main Features of the Bio-Optical Model (For the IOPs)}

[9] The MM01 model, which was designed for oceanic (case 1) waters, is thus explicitly dependent on the sole chlorophyll concentration. Therefore the input quantities are either physical constants (for pure water) or are expressed as a function of Chl. Only the main features of the model are recalled below, in reference to the description already given by MM01. The equations cited below are identified according to the same numbers that were used in MM01. The input parameters mix, besides physical constants, field results (i.e., statistical relationships) and some inevitable assumptions, as summarized below.

[10] 1. The spectral IOPs of optically pure sea water, namely the absorption, $a_{w}(\lambda)$, and scattering, $b_{w}(\lambda)$, coefficients as well as the molecular phase function are known [Pope and Fry, 1997; Morel, 1974] and used as such. The hypothetical attenuation coefficient for downward irradiance in pure water is, by approximation, expressed (equation (4) of MM01) as $K_{w}(\lambda)=a_{w}(\lambda)+(1 / 2) b_{w}(\lambda)$; its inaccurate character (actually an underestimate) has no consequence on the results, as discussed by MM01.

[11] 2. The particle scattering coefficient, $b_{p}$, and Chl as simultaneously measured at sea are related in a statistical sense (via equation (12), adopted from Loisel and Morel [1998]). The spectral dependence of this coefficient (equation (14) of MM01) is expressed as a power law, $\lambda^{v}$, where the exponent varies from -1 to 0 (when Chl increases from 0.02 to $2 \mathrm{mg} \mathrm{m}^{-3}$ ) and then becomes constant $(=0)$ for larger concentrations. The shape of the phase function of this particulate matter is also made varying with Chl but is not wavelength-dependent [see Morel et al., 2002, Figure 3]. As a result, the backscattering probability decreases from $1.2 \%$ down to $0.2 \%$ when $\mathrm{Chl}$ increases from 0.01 up to $100 \mathrm{mg} \mathrm{m}^{-3}$ (according to equation (13) of MM01) and is spectrally neutral.

[12] 3. The Chl-dependent absorption coefficient of the water body that results from the presence of all (particulate and dissolved) materials is not precisely known. Actually, the partial coefficient for the particulate fraction, $a_{p}(\lambda, \mathrm{Chl})$, is already well documented [Bricaud et al., 1998]; however, the relative proportions of colored dissolved material versus particulate material remain uncertain so that the bulk absorption coefficient (as a function of Chl) cannot be ascertained. Therefore a proxy of this inherent property, namely the attenuation coefficient for downward irradiance, $K_{d}(\lambda)$, is used instead. There are two reasons for this choice. First, an important data bank exists that includes simultaneous determinations of Chl and $K_{d}(\lambda) ; K_{d}(\lambda)$, by approximation, is assumed to be the sum of two terms, the first one due to the water itself, $K_{w}$ and the second due to all kinds of material (of biological origin) $K_{\text {bio. }}$. It has been possible to derive statistically significant relationships between $K_{\text {bio }}(\lambda)$ and Chl that express (equation (5) of MM01)

$$
K_{\text {bio }}(\lambda, \mathrm{Chl})=\chi(\lambda)[\mathrm{Chl}]^{e(\lambda)}
$$

and

$$
K_{d}(\lambda, \mathrm{Chl})=K_{\text {bio }}(\lambda, \mathrm{Chl})+K_{w}(\lambda) .
$$

[13] Secondly, by using $K_{d}(\lambda)$, there is no need to make hypotheses about the relative proportions of particle absorption and dissolved colored matter absorption, nor about the change in these proportions as a function of Chl. Indeed, the 
Table 1. Symbols and Definitions

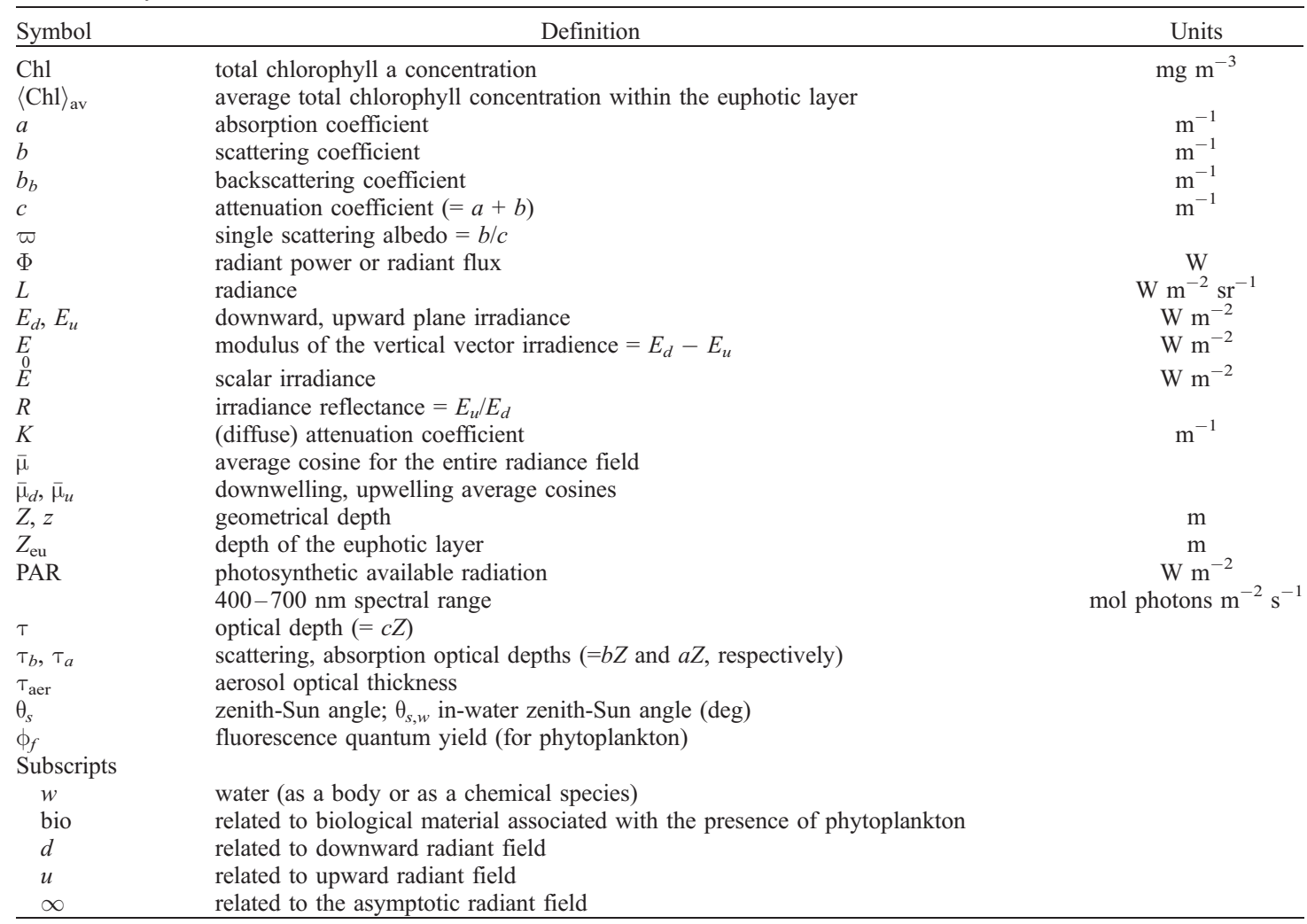

effects of both kinds of absorbers are cumulated in the formation of $K_{d}(\lambda)$.

[14] 4. The transformation of this apparent property into an inherent one, namely the absorption coefficient $a(\lambda)$, requires some iterative manipulations. They involve an exact relationship derived from the RTE (namely the "Gershun equation" with no source), yet used in an approximate fashion. The approximation originates from the adoption of "typical" values for the average cosines for the downward and upward radiant fields, $\mu_{d}$ and $\mu_{u}$, respectively (equation (8) in MM01). If $\mu_{u}$ is essentially constant (about $0.40), \mu_{d}$ is varying under the direct influence of the solar zenith angle, $\theta_{s}$ (also with the wavelength and Chl). Therefore the inversion of $K_{d}(\lambda)$ to derive $a(\lambda)$ was made under the assumption that $\theta_{s} \cong 30^{\circ}$, which represents a mean value for the experimental conditions (the $K_{d}$ determinations actually were performed at sea with $\theta_{s}$ ranging from $\sim 15^{\circ}$ to $45^{\circ}$ ). These spectral IOPs (absorption, scattering), parameterized as a function of the chlorophyll concentration, are graphically presented in Appendix A, as are some other derived quantities.

[15] 5. The last needed IOP is the Raman scattering coefficient, which is actually the fractional absorption that leads to a (transspectral) reemission by water molecules. The value adopted is $2.6 \times 10^{-4} \mathrm{~m}^{-1}$ (at $488 \mathrm{~nm}$ ), with a spectral dependency expressed as $\lambda^{-5}$ [Bartlett et al., 1998].

[16] 6 . The fluorescence by phytoplankton around $685 \mathrm{~nm}$ is another kind of emission. In order to avoid confusion between its impact upon the radiative field and that due to the Raman effect, it will be separately examined in the particular case of reflectance just beneath the surface. The way of accounting for this emission and the parameters used for its simulation are provided in Appendix B. The weak fluorescence by organic dissolved matter has a negligible impact in case 1 waters and is not considered.

\section{Computing the Underwater Radiant Field}

[17] The above model provides all the required inherent optical properties (displayed in Appendix A) to enter and operate a radiative transfer code under prescribed illumination conditions. The invariant imbedding method (HYDROLIGHT 3.0 code [Mobley, 1994]) and a Monte Carlo code [Morel and Gentili, 1996; Mobley et al., 1993] were used to numerically solve the RTE at all wavelengths between 350 and $700 \mathrm{~nm}$ (5 nm increment). The near-UV domain (350$400 \mathrm{~nm}$ ) is included because it contains radiation able to excite the Raman emission in the blue part of the spectrum. However, the results in this UV domain are not considered per se as they are obtained in a purely elastic scattering mode (without Raman sources that would be excited by still shorter wavelengths). The environmental conditions are as described by Morel et al. [2002]:

[18] 1. The spectral values of the extra terrestrial solar radiation are those proposed by Neckel and Labs [1984].

[19] 2. The barometric pressure is $1013 \mathrm{hPa}$; the aerosol is of maritime type, and its concentration corresponds to an optical thickness, $\tau_{\text {aer }}$, at $550 \mathrm{~nm}$, equal to 0.2 for systematic computations (corresponding approximately to a horizontal visibility of $30 \mathrm{~km}$ ); the $\tau_{\text {aer }}$ values 0.05 and 1 have also 
been adopted for specific cases. An overcast sky, described by a cardioidal radiance distribution, $L(\theta)=\left(L_{0} / 3\right)(1+$ $2 \cos \theta$ ), has also been considered.

[20] 3. The wind speed is zero; nevertheless, residual capillary waves persist at the sea surface, according to the formulation proposed by Cox and Munk [1955].

[21] 4. The atmosphere-ocean system is seen as a planeparallel system so that solar zenith angles $\left(\theta_{s}\right)$ exceeding $75^{\circ}$ are not considered; the standard set of solar angles comprises $0^{\circ}, 15^{\circ}, 30^{\circ}, 45^{\circ}, 60^{\circ}$, and $75^{\circ}$.

[22] 5. Preliminary computations were performed to determine the "euphotic" layer depth, $Z_{\text {eu }}$, as a function of the (uniform) chlorophyll concentration within this layer and for various $\theta_{s}$ values; $Z_{\mathrm{eu}}$ represents [Ryther, 1956] this depth, where the photosynthetically available radiation (PAR) is reduced to $1 \%$ of its value at the surface. The radiant energy PAR encompasses the whole spectrum between 400 and $700 \mathrm{~nm}$.

[23] 6. Once $Z_{\text {eu }}$ has been determined for $\mathrm{Chl}=0.03,0.1$, $0.3,1,3$, and $10 \mathrm{mg} \mathrm{m}^{-3}$, systematic computations were extended down to $3.5 Z_{\mathrm{eu}}$ and include 35 layers, each having a thickness equal to $Z_{\mathrm{eu}} / 10$ (where $Z_{\mathrm{eu}}$ is the value determined when $\theta_{s}=0^{\circ}$ ). When discussing the results, the deepest five layers were disregarded (the ascending radiant flux is misrepresented). Except if otherwise stated, the IOPs are extended without change throughout the 35 layers. Actually, such thick homogeneous layers are unrealistic most of the time, but they are convenient for the purpose of analyzing the transfer of radiation per se (including the approach of the asymptotic regime) without introducing the complexity resulting from vertical changes in the inherent properties. Nevertheless, realistic vertical nonuniform distributions will be examined for specific applications. Unrealistically, however, the emulation of the Raman emission has been in some occasions turned off, in view of quantifying its actual impact by difference.

[24] When discussing the properties for a specific layer, the adopted convention consists of using two numbers between parentheses in correspondence with the above subdivision so that the " $(0-1)$ layer" is this layer between surface and $1 / 10$ of the euphotic depth, whereas the "(910) layer" denotes this layer of thickness $Z_{\mathrm{eu}} / 10$, just above $Z_{\mathrm{eu}}$. When needed, the geometric depths are also reintroduced.

\section{Results and First Discussion}

\subsection{Sun Angle-Dependent Depth of the Euphotic Zone, $Z_{\text {eu }}$}

[25] The broadband 1\% PAR level can be established with respect to radiant power or amount of photons incident onto a surface $\left(\mathrm{Wm}^{-2}\right.$, or mole photons $\mathrm{m}^{-2} \mathrm{~s}^{-1}$, respectively). Actually, as the photosynthesis process is a quantum process, the second way is more appropriate. Also, for such studies the ${ }_{0}$ appropriate radiometric quantity is the scalar irradiance, $E$, to the extent that algal cells are able to equally capture radiant energy from all directions. Because of instrumental limitations, however, the (planar) downward irradiance, $E_{d}$, has been the most currently measured quantity at sea. In what follows the results concerning the "standard $Z_{\text {eu }}$ " refer to downward irradiance, expressed in radiometric units and for the spectral band $400-700 \mathrm{~nm}$.
Table 2. Depth of the Euphotic Layer For Various Chlorophyll Concentrations and Sun-Zenith Angles ${ }^{\mathrm{a}}$

\begin{tabular}{|c|c|c|c|c|c|c|}
\hline \multirow[b]{2}{*}{$\theta_{s}, \operatorname{deg}$} & \multicolumn{6}{|c|}{$\mathrm{Chl}, \mathrm{mg} \mathrm{m}^{-3}$} \\
\hline & 0.03 & 0.1 & 0.3 & 1.0 & 3.0 & 10.0 \\
\hline \multicolumn{7}{|c|}{$A$} \\
\hline 0 & 178.6 & 113.2 & 69.7 & 39.7 & 23.4 & 12.6 \\
\hline 15 & 176.5 & 112.0 & 69.0 & 39.3 & 23.2 & 12.5 \\
\hline 30 & 168.2 & 106.8 & 65.9 & 37.7 & 22.3 & 12.0 \\
\hline 45 & 159.7 & 101.5 & 62.9 & 36.1 & 21.4 & 11.6 \\
\hline 60 & 148.3 & 94.5 & 58.7 & 33.8 & 20.2 & 11.0 \\
\hline 75 & 145.4 & 92.5 & 57.4 & 33.1 & 19.7 & 10.8 \\
\hline Overcast & 166.0 & 105.7 & 65.2 & 37.2 & 22.0 & 11.9 \\
\hline \multicolumn{7}{|c|}{$B$} \\
\hline 0 & 129.5 & 99.9 & 59.4 & 37.7 & 23.5 & 13.5 \\
\hline 15 & 128.6 & 88.2 & 58.9 & 37.3 & 23.3 & 13.3 \\
\hline 30 & 124.7 & 85.2 & 56.7 & 35.9 & 22.5 & 12.9 \\
\hline 45 & 120.6 & 82.1 & 54.5 & 34.4 & 21.6 & 12.4 \\
\hline 60 & 115.1 & 77.8 & 51.4 & 32.4 & 20.4 & 11.7 \\
\hline 75 & 113.5 & 76.6 & 50.4 & 31.7 & 19.9 & 11.4 \\
\hline \multicolumn{7}{|c|}{ C } \\
\hline 0 & 169.2 & 108.3 & 67.4 & 38.9 & 23.2 & 12.6 \\
\hline 15 & 167.2 & 107.1 & 66.7 & 38.5 & 23.0 & 12.5 \\
\hline 30 & 159.2 & 102.0 & 63.7 & 36.9 & 22.1 & 12.0 \\
\hline 45 & 150.9 & 96.9 & 60.7 & 35.3 & 21.2 & 11.6 \\
\hline 60 & 139.7 & 89.8 & 56.5 & 33.1 & 20.0 & 11.0 \\
\hline 75 & 136.6 & 87.8 & 55.2 & 32.3 & 19.5 & 10.7 \\
\hline \multicolumn{7}{|c|}{$D$} \\
\hline 0 & 188.1 & 110.2 & 73.4 & 41.9 & 28.9 & 13.5 \\
\hline 15 & 185.7 & 117.7 & 72.5 & 41.4 & 24.6 & 13.3 \\
\hline 30 & 175.7 & 111.3 & 68.7 & 39.3 & 23.4 & 12.7 \\
\hline 45 & 165.2 & 104.8 & 64.7 & 37.2 & 22.2 & 12.1 \\
\hline 60 & 150.4 & 95.4 & 59.1 & 34.1 & 20.5 & 11.2 \\
\hline 75 & 145.0 & 91.9 & 57.0 & 32.8 & 19.7 & 10.8 \\
\hline \multicolumn{7}{|c|}{$E$} \\
\hline 0 & 178.8 & 114.3 & 71.1 & 41.1 & 24.7 & 13.5 \\
\hline 15 & 176.4 & 112.8 & 70.2 & 40.6 & 24.4 & 13.4 \\
\hline 30 & 166.7 & 106.6 & 66.4 & 38.6 & 23.2 & 12.8 \\
\hline 45 & 156.5 & 100.1 & 62.6 & 36.4 & 22.0 & 12.1 \\
\hline 60 & 141.9 & 90.9 & 57.0 & 33.3 & 20.2 & 11.2 \\
\hline 75 & 136.4 & 87.3 & 54.8 & 32.1 & 19.4 & 10.8 \\
\hline
\end{tabular}

${ }^{\mathrm{a}}$ Depth of the euphotic layer $\left(Z_{\mathrm{eu}}, \mathrm{m}\right)$ as a function of the chlorophyll concentration $\left(\mathrm{mg} \mathrm{m}^{-3}\right)$ and the zenith-Sun angle (deg), or, on one occasion, for an overcast sky. A, uniform chlorophyll profiles and $Z_{\mathrm{eu}}$ defined on the basis of downward irradiance, $E_{d}$, expressed in radiometric units $\left(\mathrm{W} \mathrm{m}^{-2}\right)$; $\mathrm{B}$, as in A, but for nonuniform chlorophyll profiles (see text); $\mathrm{C}$, as in A, but when $E_{d}$ is expressed in quantum units (mol photons $\mathrm{m}^{-2} \mathrm{~s}^{-1}$ ); $\mathrm{D}$, as in A, but when the definition of $Z_{\mathrm{eu}}$ is based on scalar irradiance; $\mathrm{E}$, as in $\mathrm{D}$, but when the scalar irradiance is expressed in quantum units (mol photons $\mathrm{m}^{-2} \mathrm{~s}^{-1}$ ).

The changes in $Z_{\text {eu }}$, when scalar irradiance and (or) quantum units are preferred, will be examined as deviations with respect to these standard $Z_{\text {eu }}$ values. Numerical results are provided in Table 2 .

[26] The first set of computations deals with uniform Chl profiles $\left(\mathrm{Chl}=0.03,0.1,0.3,1,3\right.$, and $\left.10 \mathrm{mg} \mathrm{m}^{-3}\right)$ and $\theta_{s}=0^{\circ}, 15^{\circ}, 30^{\circ}, 45^{\circ}, 60^{\circ}$, and $75^{\circ}$. The $Z_{\text {eu }}$ values presently obtained (upper part of Table 2) as a function of the mean chlorophyll concentration are close to those previously determined through the static approach used in MM01 (Figure 1). They also bracket the statistical relationship [Morel, 1988] that was directly derived from field data and based on simultaneous determinations of $Z_{\text {eu }}$ and the mean $\mathrm{Chl}$ concentration, $\langle\mathrm{Chl}\rangle$, within the euphotic layer. This overall agreement with "sea truth" measure- 


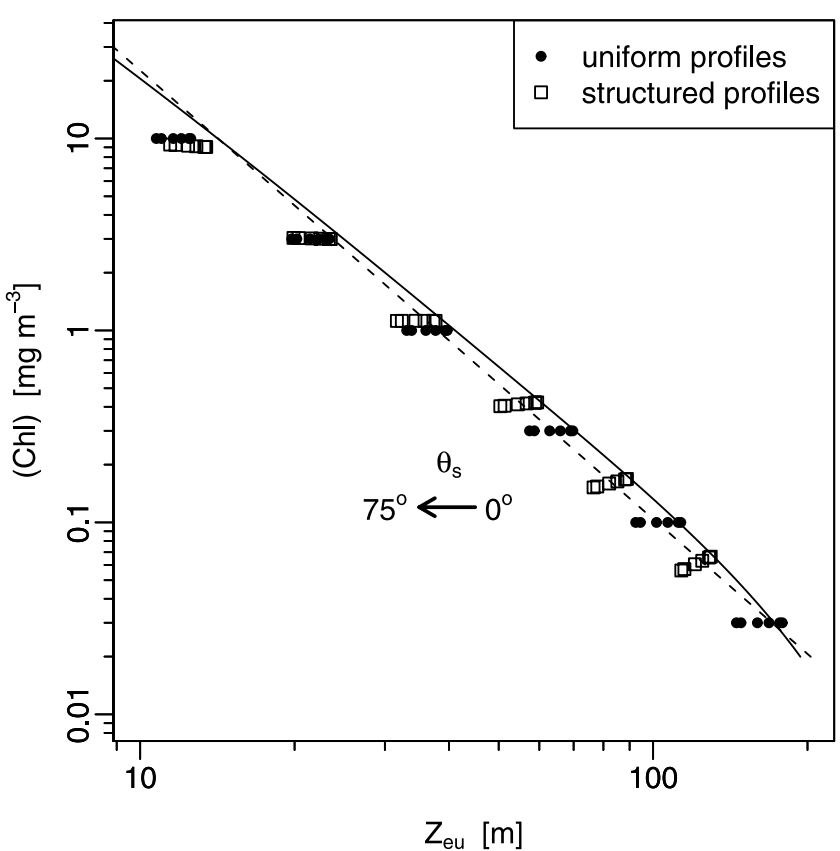

Figure 1. Depth of the euphotic layer, defined in terms of $E_{d}$ and energy (within the PAR domain), as a function of $\langle\mathrm{Chl}\rangle$, the mean chlorophyll concentration within the euphotic layer. The solid symbols are for uniform Chl profiles, and the open symbols are for structured nonuniform profiles (see text). The symbols, from right to left, correspond to increasing Sun-zenith angle $\left(\theta_{s}\right.$ from $0^{\circ}$ up to $75^{\circ}$ ). In the case of structured profiles (including a deep chlorophyll maximum), the decrease in $Z_{\text {eu }}$, when $\theta_{s}$ increases, leads to a correlative decrease in the mean concentration (at least noticeable in low-Chl oligotrophic waters). The dashed line represents the empirical relationship [Morel, 1988], namely $Z_{\mathrm{eu}}=38$ [Chl] $^{-0.428}$, whereas the solid line reproduces the curve presented by MM01 (their Figure 6).

ments is reassuring about the validity of the input parameters used in the full RTE computations. Indeed, in the previous purely empirical approaches, parameters like the scattering coefficient and the particle VSF were not

Figure 2. (a) Ratio of $Z_{\mathrm{eu}}\left(\theta_{s}\right)$ to $Z_{\mathrm{eu}}$ when $\theta_{s}=0$, plotted as a function of the cosine of the refracted solar angle $\left(\cos \theta_{s, w}\right)$, for various chlorophyll concentrations as indicated, and when the chlorophyll profiles are uniform. The solid curves are for a clear sky with an aerosol optical thickness $\tau_{\text {aer }}=0.2$; the dashed curves are for a hazy sky $\left(\tau_{\text {aer }}=1\right)$; the isolated closed symbols are for an overcast sky with a cardioidal radiance distribution; their abscissae correspond to the $\bar{\mu}_{d}$ value, as calculated just below the surface. (b) As in Figure 2a, and only with $\tau_{\text {aer }}=0.2$ for uniform chlorophyll profiles (dashed lines, reproduced from Figure 2a) and for structured profiles (solid lines) with the same chlorophyll concentration in the surface layer and modeled as by Morel and Berthon [1989]. Note that the ordering of the curves with respect to the chlorophyll concentration is modified for the structured profiles, compared to uniform profiles (actually, uniform and nonuniform profiles are almost coinciding for high Chl, whereas they strongly differ for low $\mathrm{Chl}$ ). involved at all, whereas they are now present in the RTE computations.

[27] The $Z_{\text {eu }}$ values are, as expected, dependent on the Sun angle, and the decrease in $Z_{\mathrm{eu}}$ when $\theta_{s}$ increases from $0^{\circ}$ to $75^{\circ}$ is of the order $14-18 \%$ (Figure $2 \mathrm{a}$ ). Were the water a purely absorbing medium (and the Sun in a black sky), the decrease in $Z_{\text {eu }}$ would exactly follow that of $\mu_{s w}$, the cosine of the solar angle, after refraction (dashed line in Figure 2). Actually, the $Z_{\mathrm{eu}}$ decrease is much less pronounced as it is thwarted by two phenomena: first, the rearrangement of the light field due to scattering and second, the effect of the diffuse sky radiation. The first effect is the most important, as evidenced by the divergence between the dashed line and
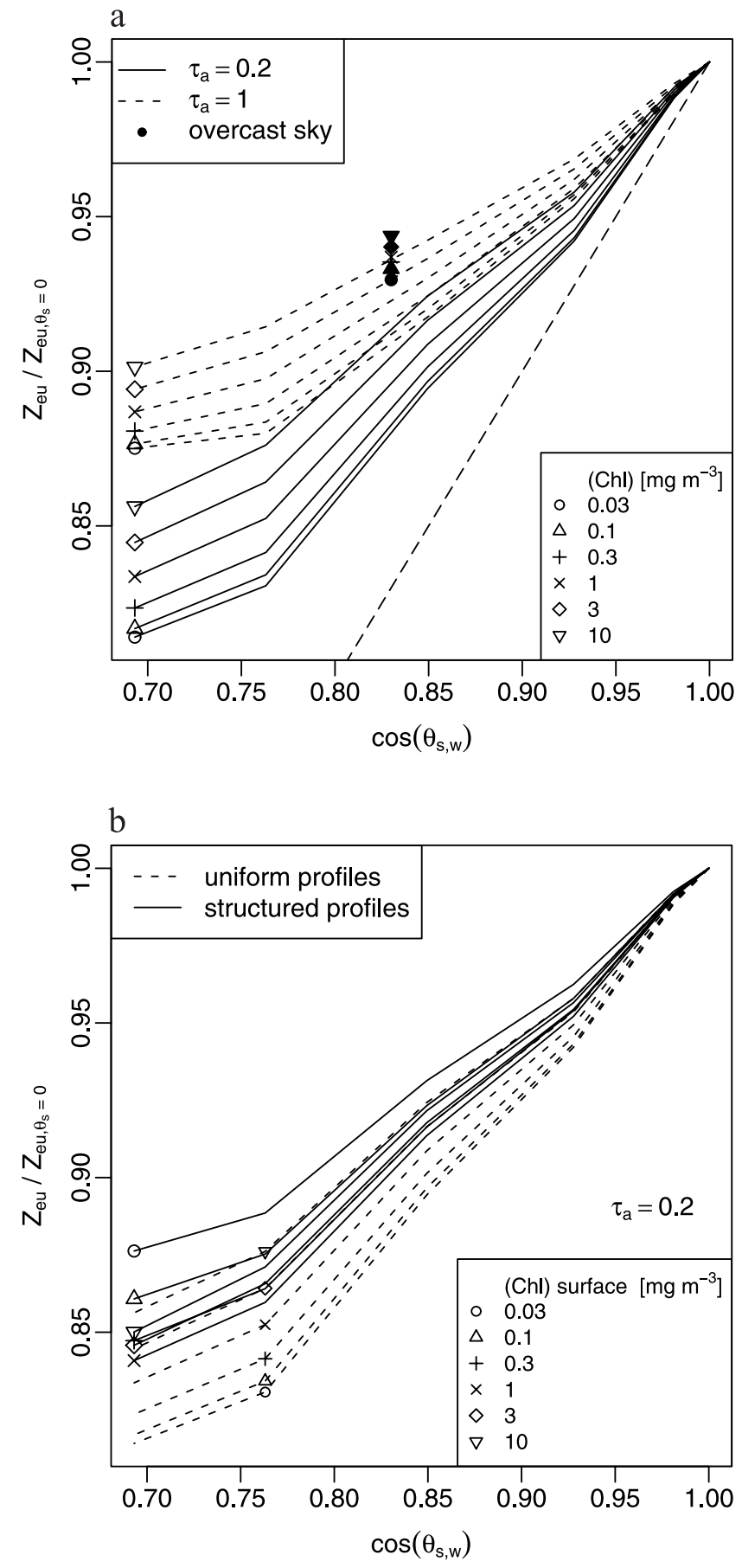
those showing the actual decreases in $Z_{\text {eu }}$; this is also clearly seen (Figure 2a) when $\mathrm{Chl}$ is allowed to increase from 0.03 to $10 \mathrm{mg} \mathrm{m}^{-3}$, which leads to an increase in scattering and, more importantly, in the single scattering albedo $\varpi=b / c$ (see Appendix A). The second effect is put in evidence by changing the atmospheric conditions from standard (clear, with $\left.\tau_{\text {aer }}=0.2\right)$ to bright hazy $\left(\tau_{\text {aer }}=1\right)$ and to fully overcast skies (Figure 2a).

[28] A second set of computations deals with nonuniform pigment profiles, which include the presence of a deep chlorophyll maximum (DCM). The DCM is particularly developed in stratified oligotrophic waters, whereas it practically vanishes when Chl exceeds $1 \mathrm{mg} \mathrm{m}^{-3}$. These profiles are modeled as by Morel and Berthon [1989] as a function of the surface layer $\mathrm{Chl}$ value and include a Gaussian-shaped DCM. With the same concentration near the surface (for uniform or nonuniform profiles) the average concentration within the whole euphotic layer, denoted $\langle\mathrm{Chl}\rangle_{\mathrm{av}}$, is increased in nonuniform profiles to the extent that the DCM is (at least partly) included within this layer. For instance, with $\mathrm{Chl}=0.03 \mathrm{mg} \mathrm{m}^{-3}$ at the surface, $\langle\mathrm{Chl}\rangle_{\mathrm{av}}$ is about $0.08 \mathrm{mg} \mathrm{m}^{-3}$ for nonuniform ("structured") profiles; $\langle\mathrm{Chl}\rangle_{\text {av }}$ decreases when $Z_{\text {eu }}$ decreases under the effect of increasing solar angle (see Figure 1). The presence of the DCM and thus the correlative increase of the mean concentration obviously entails an ascent of the $Z_{\text {eu }}$ depth, which is particularly marked for oligotophic waters. Nevertheless, the prediction of $Z_{\mathrm{eu}}$ from $\langle\mathrm{Chl}\rangle_{\mathrm{av}}$ is not appreciably different from the one obtained with a uniform profile, provided that $\mathrm{Chl}$ is given the appropriate $\langle\mathrm{Chl}\rangle_{\mathrm{av}}$ value. Even if, in principle, the shape of the pigment profile and the position of the maximum must affect the result, this effect remains minor so that the dependence of $Z_{\mathrm{eu}}$ on $\theta_{s}$ is essentially the same as for uniform profiles with an equivalent $\left(\mathrm{Chl}=\langle\mathrm{Chl}\rangle_{\mathrm{av}}\right)$ concentration. The changes in $Z_{\mathrm{eu}}$ with solar angle for structured profiles are shown in Figure $2 b$.

\subsection{Depth of the Euphotic Zone According to Various Definitions (Table 2)}

[29] The consequences of the various definitions of $Z_{\text {eu }}$ can be briefly analyzed. If the $1 \%$ level is defined in terms of amount of photons, a spectral effect is expected. In blue waters (low Chl), less photons than in green waters (high $\mathrm{Chl}$ ) are needed to produce the same energy (indeed, the quanta-to-Joule ratio varies between $2.3 \times 10^{18}$ and $2.7 \times$ $10^{18}$ when Chl varies from 0.03 up to $10 \mathrm{mg} \mathrm{m}^{-3}$ [Morel and Smith, 1974]). Accordingly, $Z_{\mathrm{eu}}^{Q}$ is smaller than $Z_{\mathrm{eu}}^{W}$ (superscripts $Q$ and $W$ for quanta and watt, respectively) by about $5 \%$ when $\mathrm{Chl}=0.03 \mathrm{mg} \mathrm{m}^{-3}$. This negative difference, which is practically independent from the Sun angle, regularly decreases when $\mathrm{Chl}$ increases and finally annihilates at $10 \mathrm{mg} \mathrm{m}^{-3}$.

[30] If $Z_{\text {eu }}^{W}$ refers to scalar irradiance $(\stackrel{0}{E})$ instead of downward irradiance $\left(E_{d}\right)$, a purely geometrical Sun-related effect is involved and is almost independent from the chlorophyll concentration). $Z_{\mathrm{eu}}^{W}(\stackrel{0}{E})$ is deeper than $Z_{\mathrm{eu}}^{W}\left(E_{d}\right)$ by about $6 \%$ when $\theta_{s}=0^{\circ}$, whereas this positive difference vanishes for $\theta_{s}=75^{\circ}$. If both modifications in the definition are cumulated, namely if $Z_{\mathrm{eu}}$ is defined in terms of photons and scalar irradiance instead of in terms of energy and downward irradiance, the resulting differences in terms of geometrical depths are minute. Indeed, the opposite trends

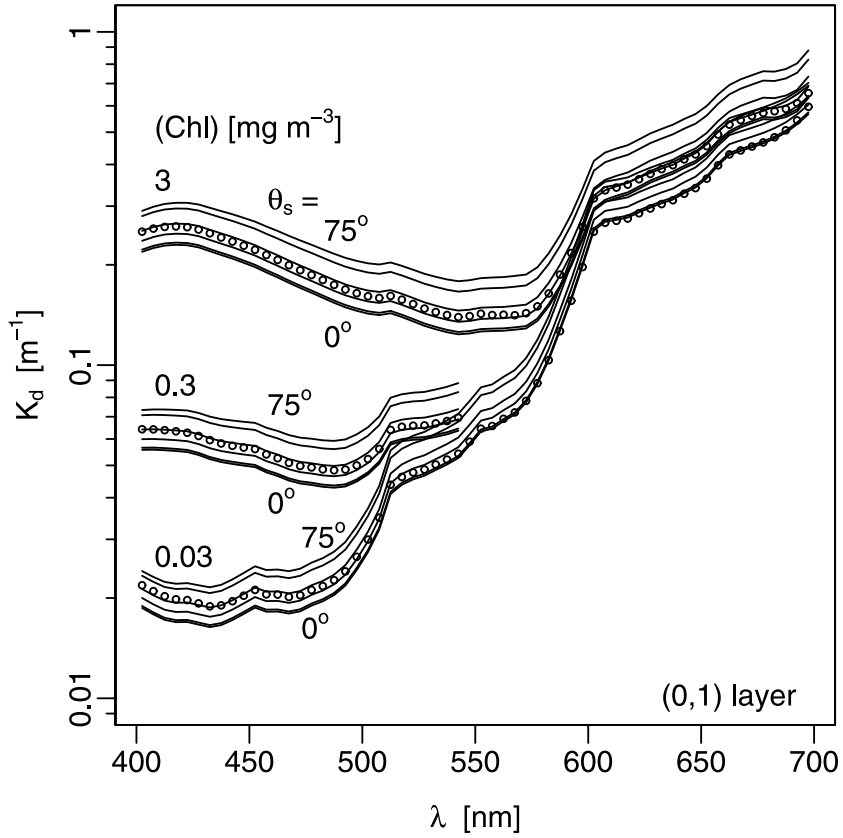

Figure 3. Spectral $K_{d}$ values for the upper layer, three selected chlorophyll concentrations, as indicated, and for increasing zenith Sun angle $\left(0^{\circ}-75^{\circ}\right)$ from bottom to top. For the sake of clarity, the values when $\mathrm{Chl}=0.3 \mathrm{mg} \mathrm{m}^{-3}$ are not fully displayed. Also shown (open symbols) are the spectra obtained for the same Chl values by using equation (2) and Table 2 in MM01.

mentioned above lead to an approximate compensation. Therefore the question of a precise and realistic definition of the euphotic depth, which is sound in its principle, appears in practice to be rather academic. The predictive skill of models as well as the experimental accuracy are not such that the small expected differences can be indisputably put in evidence or have a real impact. In contrast, the significant changes in $Z_{\text {eu }}$ with $\theta_{s}$ have to be taken into account, which can be simply achieved by using the dependence upon $\mu_{0, w}$, as suggested by Figures $2 \mathrm{a}$ and $2 \mathrm{~b}$.

[31] Although the actual depth of the euphotic layer does not depend significantly on the definition which is adopted, only the use of scalar irradiance is appropriate when dealing with absorption (by phytoplankton for instance for photosynthesis studies, or globally when assessing the heating rate). Indeed, the amount of locally absorbed radiation is exactly expressed as the product $a E$, and $E_{d}$ is always an underestimate of ${ }_{E}^{0}$. Therefore a comparison between $E$ and $E_{d}$ is to be made. This comparison will be displayed later on as its interpretation requires the knowledge of several other parameters of the radiant field, to be examined first.

\subsection{Attenuation Coefficient of Downwelling Irradiance $\left(K_{d}\right)$ Within the Upper Layer}

[32] The upper layer considered here has a thickness corresponding to $1 / 10$ of the euphotic layer (when $\theta_{s}=0$ ), and the $K_{d}(\lambda)$ values are the mean values computed for the $(0-1)$ depth interval. Some examples resulting from the present analytical approach are displayed in Figure 3. The increase in all $K_{d}(\lambda)$ values when the solar angle increases is significant (by $35-45 \%$ when $\theta_{s}$ varies from 


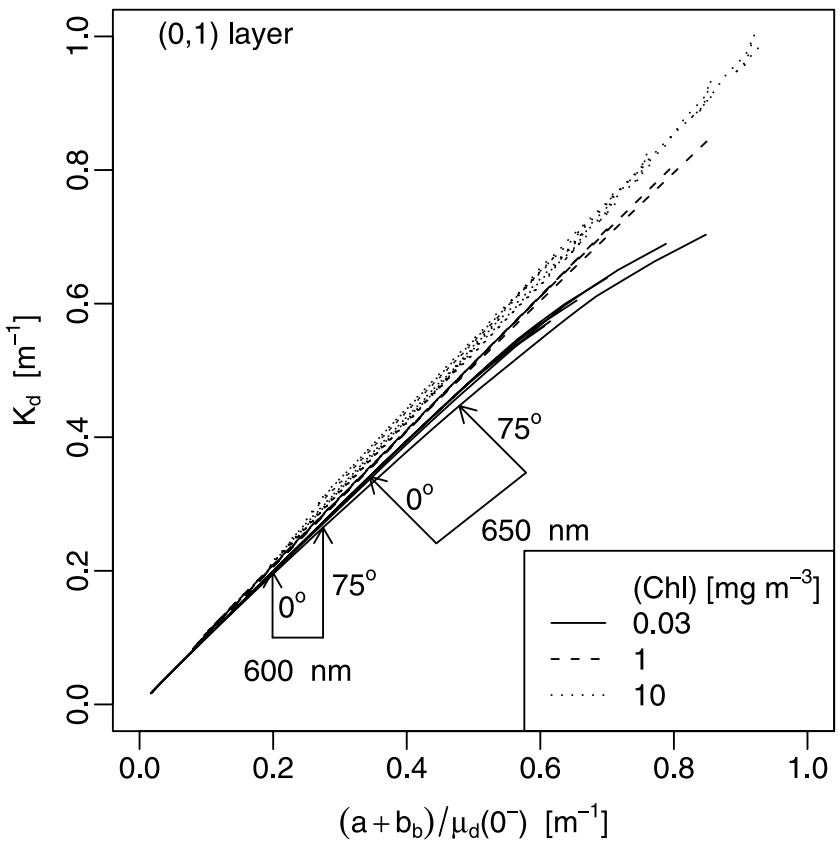

Figure 4. For three selected Chl values, all spectral $K_{d}$ values for all $\theta_{s}$ values and for the first upper layer, plotted as a function of the quantity $\left(a+b_{b}\right) / \bar{\mu}_{d}$, where $\bar{\mu}_{d}$ stands for the average cosine for the downward irradiance computed just beneath the surface (equation (2)). Note that when $\mathrm{Chl}=10 \mathrm{mg} \mathrm{m}^{-3}$, the mean slope is about 1.045 , and about 1.00 when $\mathrm{Chl}=1 \mathrm{mg} \mathrm{m}^{-3}$. When $\mathrm{Chl}=0.03 \mathrm{mg} \mathrm{m}^{-3}$, a departure from linearity (arrow) occurs for the largest $K_{d}$ values found in the red part of the spectrum and denotes the impact of the Raman emission.

$0^{\circ}$ to $75^{\circ}$ ) and practically independent from the wavelength. As expected, it is ruled by the correlative decrease in the average cosine for downward irradiance beneath the surface $\mu_{d}$ and is written as [Gordon, 1989]

$$
K_{d}=1.0395\left(\bar{\mu}_{d}\right)^{-1}\left(a+b_{b}\right) .
$$

Note that this (approximate) formula has been established for a $K_{d}$ value just beneath the surface, i.e., as a limit of $K_{d}$ when the thickness of the upper layer tends toward zero.

[33] These spectra can be compared to those produced by the Sun-independent MM01 model. Recall that this model resulted from a straightforward use of the statistical analysis between $\left(K_{d}-K_{w}\right)$ and [Chl] (equation (1a)). This comparison is provided by Figure 3 , where the spectral $K_{d}$ values from the MM01 model are superimposed onto the corresponding values produced by the radiative transfer model for varying solar angles. The overall agreement is excellent and remains as such for other Chl values (not shown).

[34] Actually, the spectra obtained through equations (1a) and (1b) coincide best with the analytical ones for $\theta_{s} \cong$ $30^{\circ}-40^{\circ}$. This is consistent with the fact that the absorption coefficient has been iteratively derived from experimental $K_{d}$ by assuming a Sun angle of about $30^{\circ}$ (see above). The agreement is also comforting with regard to the validity of the other IOPs introduced into the present computations (namely the particle scattering coefficient and phase function).

[35] The approximate relationship (equation (2)) between $K_{d}$ and the IOPs, $a$ and $b_{b}$, is illustrated by Figure 4 . When $\mathrm{Chl}=0.03 \mathrm{mg} \mathrm{m}^{-3}$, a departure from linearity occurs for the highest $K_{d}$ values, which actually belong only to the red part of the spectrum. This departure denotes the depressive impact of the Raman emission on $K_{d}$, which progressively fades for increasing $\mathrm{Chl}$ and is no longer distinguishable when $\mathrm{Chl}=1 \mathrm{mg} \mathrm{m}^{-3}$. At greater depths the situation is quite different (see below).

\section{4. $K_{d}$ Evolution With Depth}

[36] The results shown (Figures 5a and 5b) and discussed below refer to successive layers, as indicated. For low Chl $\left(\leq 0.3 \mathrm{mg} \mathrm{m}^{-3}\right)$ a drastic change quickly affects the red part of the spectrum. In the shallow (2-3) layer the $K_{d}(\lambda)$ values for $\lambda>600 \mathrm{~nm}$ are markedly depressed under the influence of the Raman emission, which already predominates at such depths over the transmitted light. For the deepest layer, just above $Z_{\text {eu }}$, there is no longer any transmitted red light; it is totally replaced by the emitted radiation so that the $K_{d}$ spectrum between 580 and $700 \mathrm{~nm}$ actually reproduces the shape of the $K_{d}$ spectrum between 490 and $570 \mathrm{~nm}$, i.e., that of the exciting radiation (see also the $K_{d}$ spectrum computed for the asymptotic regime by Gordon et al. [1993, Figure 15]).

[37] By contrast, when considering the situation with $\mathrm{Chl}=10 \mathrm{mg} \mathrm{m}^{-3}$, the Raman radiation has no palpable influence (except close to $700 \mathrm{~nm}$ ) on the shape of the $K_{d}$ spectrum, even for the deepest layer shown (9-10); nevertheless, the Raman influence shows itself deeper. The situation for $\mathrm{Chl}=10 \mathrm{mg} \mathrm{m}^{-3}$ is rather unique (Figure $5 \mathrm{~b}$ ). Indeed, even when $\mathrm{Chl}$ is as high as $3 \mathrm{mg} \mathrm{m}^{-3}$, the Raman influence within the deepest layer is detectable beyond $600 \mathrm{~nm}$ and is marked beyond $650 \mathrm{~nm}$. It is also worth noting that within this deep layer the spectra tend to become almost independent from the Sun position, which suggests that a diffuse quasi-asymptotic regime would be approached for all the wavelengths (but see later for a closer examination).

\subsection{Attenuation Coefficient of Upwelling Irradiance $\left(K_{u}\right)$}

[38] The $K_{u}(\lambda)$ values computed for the same upper (0 $\left.Z_{\text {eu }} / 10\right)$ layer as above are displayed in Figure $6 a$ and, for deeper layers, in Figure 6b. Compared to the $K_{d}(\lambda)$ spectra, the striking difference for the upper layer occurs for the red part of the $K_{u}(\lambda)$ spectrum under the influence of the Raman emission (compare with Figure 3). The depressive effect, which is maximal when $\mathrm{Chl}=0.03 \mathrm{mg} \mathrm{m}^{-3}$, progressively weakens when Chl increases; still detectable for $1 \mathrm{mg} \mathrm{m}^{-3}$, it disappears for $10 \mathrm{mg} \mathrm{m}^{-3}$. For this concentration, it can be noticed that the $K_{u}(\lambda)$ variations with respect to the solar position have a greater amplitude than those for $K_{d}(\lambda)$, which is rather counterintuitive; also, the minimal $K_{u}$ values in the green part of the spectrum (around $560 \mathrm{~nm}$ ) are distinctly below the corresponding $K_{d}$ values.

[39] In all cases the inequality $K_{u}(\lambda)<K_{d}(\lambda)$ is always satisfied, either in the inelastic (Raman) scattering domain or in the elastic domain $(\lambda<570 \mathrm{~nm})$. A convenient way to put the inequality in evidence is to form the normalized difference, i.e., the quantity $\left(K_{d}-K_{u}\right) / K_{d}$. There are some 

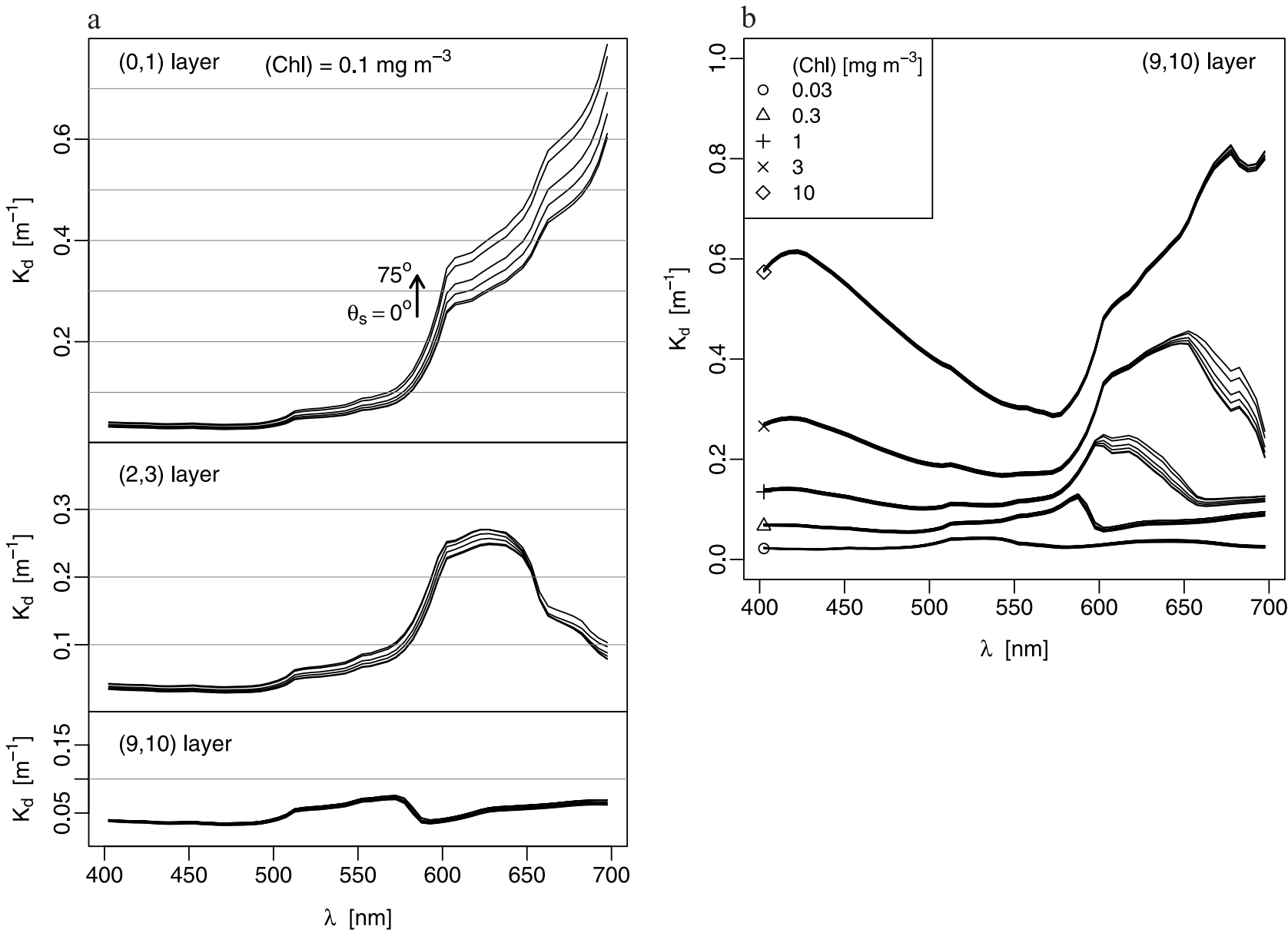

Figure 5. (a) For $\mathrm{Chl}=0.1 \mathrm{mg} \mathrm{m}^{-3}$, the $K_{d}$ spectra for the upper layer, an intermediate layer, and at the level of the euphotic depth, from top to bottom, respectively. In each panel, the various curves correspond to the six solar zenith angle $\left(\theta_{s}\right.$ values). For the upper layer, the spectra would be parallel if plotted according to a log scale. (b) For the other Chl values, the $K_{d}$ spectra at the level of the last layer, just above the euphotic depth.

practical applications of this representation (see later) and also a physical basis as the difference equates to the relative gradient of reflectance with respect to depth, according to the identity (itself deriving from the definitions)

$$
\left(K_{d}-K_{u}\right) \equiv(1 / R)(d R / d z)
$$

In a nonconservative $(\varpi<1)$ homogeneous medium, this relative gradient is strictly null when the asymptotic regime is reached; otherwise, it is generally positive. Under slant illumination conditions, it might be negative (see Figure 10). Therefore the quantity $\left(K_{d}-K_{u}\right) / K_{d}$ most often remains within the $[0-1]$ domain but may be slightly negative for large $\theta_{s}$ values. It is also worth noting that this quantity is always decreasing for increasing $\theta_{s}$ values.

[40] Instances of this normalized difference are provided in Figure 7 (upper panels for the upper layer). For $\mathrm{Chl}=$ $0.03 \mathrm{mg} \mathrm{m}^{-3}$ the relative difference is near zero $(<10 \%)$ in the blue part of the spectrum, increases slowly beyond $520 \mathrm{~nm}$, and then increases abruptly beyond $570 \mathrm{~nm}$ and exceeds 50\% from 600 to $700 \mathrm{~nm}$ because the Raman emission provides a significant contribution to the upward stream, even near the surface. Therefore the rate of its decrease along the depth is lower than the rate that regulates the downward stream essentially made of elastically transmitted (red) radiation; as a consequence, $K_{d}$ largely exceeds $K_{u}$. With increasing depth (Figure 7; see also Figure 6b), when the downward radiant flux is also exclusively made up of emitted radiation, the relative difference returns to zero in the red, while a "bump" forms and migrates toward the green part of the spectrum; actually, this bump vanishes at a depth larger than twice $Z_{\text {eu }}$.

[41] For high Chl $\left(10 \mathrm{mg} \mathrm{m}^{-3}\right)$, and for the first optical layer, the situation is quite different, with a strong effect of the Sun position upon the relative difference, an absence of Raman effect, and a maximal deviation (about 50\%) in the green part of the spectrum (around $570 \mathrm{~nm}$, in keeping with the absorption minimum and thus with the reflectance maximum). With increasing depth, the Raman feature appears in the far red as a steep increase; then, deeper, it also creates a bump migrating toward the shorter wavelengths.

[42] In effect, this bump is an interesting feature or indicator. On the short-wavelength side the null value of the normalized difference means that a quasi-asymptotic regime is being reached for the elastically transmitted 
a

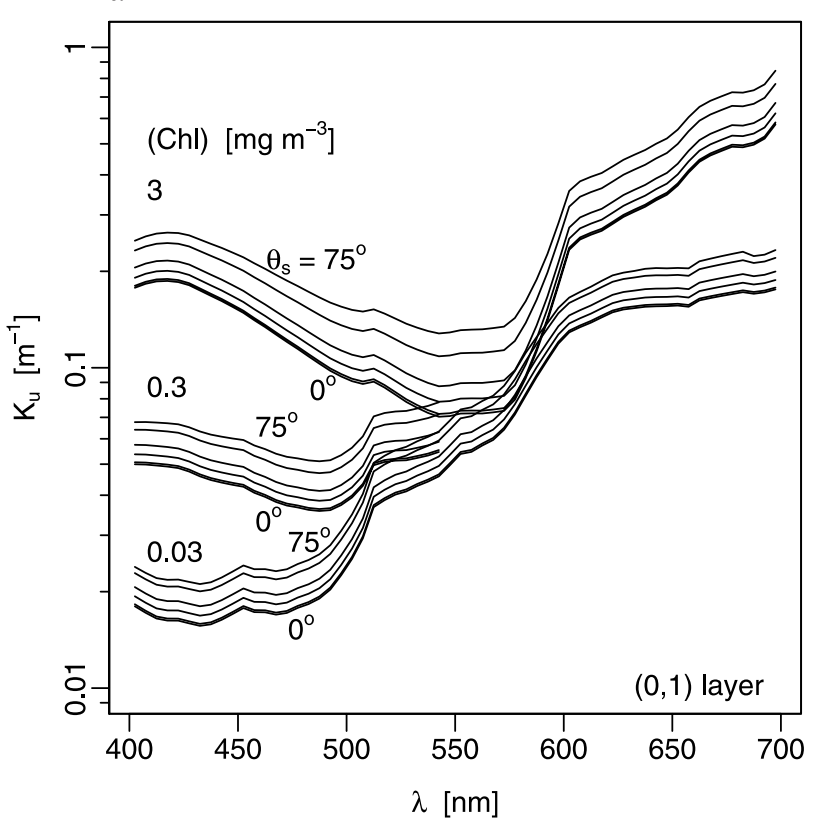

b

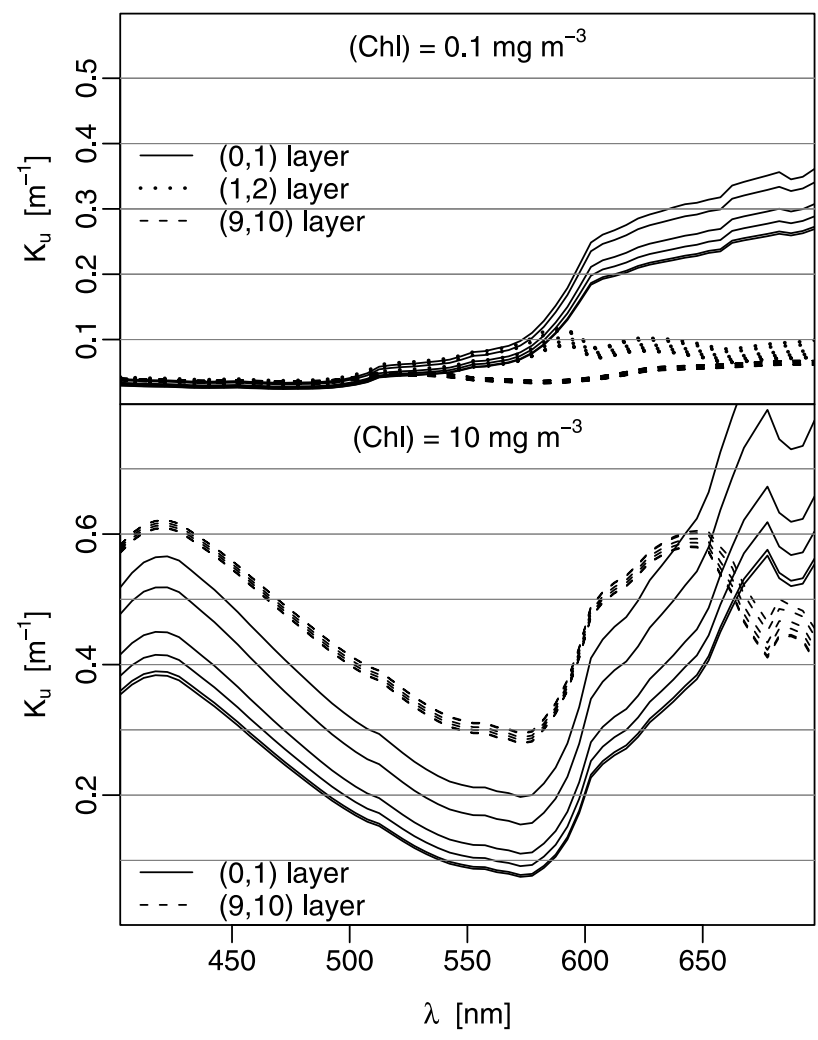

Figure 6. (a) As in Figure 3, but for $K_{u}$ instead of $K_{d}$. (b) $K_{u}$ spectra for Chl $=0.1$ and $10 \mathrm{mg} \mathrm{m}^{-3}$, and various layers, as indicated.

radiation; on the long-wavelength side the null value means that the radiative field is entirely built up with Raman emission. Actually, the bump itself marks the narrow spectral domain where elastic and inelastic scattered radiation still coexist and are unbalanced between the downward and the upward streams.

\subsection{Spectral Reflectance Just Beneath the Surface}

[43] The behavior of the irradiance reflectance $R\left(\lambda, \theta_{s}\right.$, $\mathrm{Chl}, 0^{-}$) just below the surface has already been analyzed in a previous paper dealing with the bidirectional effects [Morel et al., 2002], which was based on the same RTE computations and IOPs model as those used here. In correspondence with typical channels of ocean color sensors, only seven wavelengths were selected and studied, whereas the whole spectra are now shown. The variation in $R$ with a varying solar angle is simply expressed through a factor $\mathrm{f}$, which mainly depends on the three quantities $\theta_{s}, \lambda$, and Chl (and secondarily on $\tau_{\text {aer }}$ and the wind speed, not made explicit here):

$$
R\left(\theta_{s}, \lambda, \mathrm{Chl}, 0^{-}\right)=f\left(\theta_{s}, \lambda, \mathrm{Chl}\right)\left[b_{b}(\lambda, \mathrm{Chl}) / a(\lambda, \mathrm{Chl})\right] .
$$

Whatever the wavelength and chlorophyll concentration, the $f\left(\theta_{s}, \lambda, \mathrm{Chl}\right)$ factor (and thus $\left.R\right)$ takes its minimal value, $f(0$, $\lambda, \mathrm{Chl}$ ), when $\theta_{s}=0$, with values between $\sim 0.30$ and 0.42 [see Morel et al., 2002, Figure 7a]; then, with increasing $\theta_{s}$ values, $f$ systematically increases, in various manners, however, depending on the $\lambda$ and Chl values. The very causes of these variations in $f$, which have been previously explained [Morel et al., 2002; Morel and Gentili, 1996], are not repeated here.

[44] For a given $(\lambda-\mathrm{Chl})$ couple the evolution is yet rather simple as $f$ is to a good approximation linearly related to $\left(1-\cos \theta_{s}\right)$. Parameterizations of these evolutions have been proposed [Morel et al., 2002, Figures 7-8, Table 1] and are of the form

$$
f\left(\theta_{s}, \lambda, \mathrm{Chl}\right)=f(0, \lambda, \mathrm{Chl})+S_{f}(\lambda, \mathrm{Chl})\left(1-\cos \theta_{s}\right) .
$$

The Sun angle effect upon the entire $R$ spectra (just beneath the surface) can be seen in Figure 8a. The enhancement of the reflectance values (when the solar zenith angle is increasing) is increasingly important from low Chl to high Chl (which means that for a given $\lambda$ value the slope parameter, $S_{f}$, is always increasing with the Chl value). In other words, the sensitivity to the Sun position and the magnitude of the change in $R(\lambda)$ are more marked for waters with a high chlorophyll content. When $\mathrm{Chl}=10 \mathrm{mg} \mathrm{m}^{-3}$, for instance, the $R(\lambda)$ values are almost doubled when $\theta_{s}$ increases from $0^{\circ}$ to $75^{\circ}$. The relative enhancement is not spectrally neutral; the $\left[S_{f}(\lambda, \mathrm{Chl})\right]$ term in equation (4b) increases from the blue to the red part of the spectrum (when $\mathrm{Chl} \leq 1 \mathrm{mg} \mathrm{m}^{-3}$ ), or it passes through a maximum in the green part of the spectrum $(560-570 \mathrm{~nm})$, when $\mathrm{Chl}>$ $1 \mathrm{mg} \mathrm{m}^{-3}$. Therefore in their evolution with $\theta_{s}$ the shapes of the $R(\lambda)$ spectra are slightly modified (a phenomenon not yet accounted for in the development of algorithms for ocean color processing and considered as insignificant in MM01). 


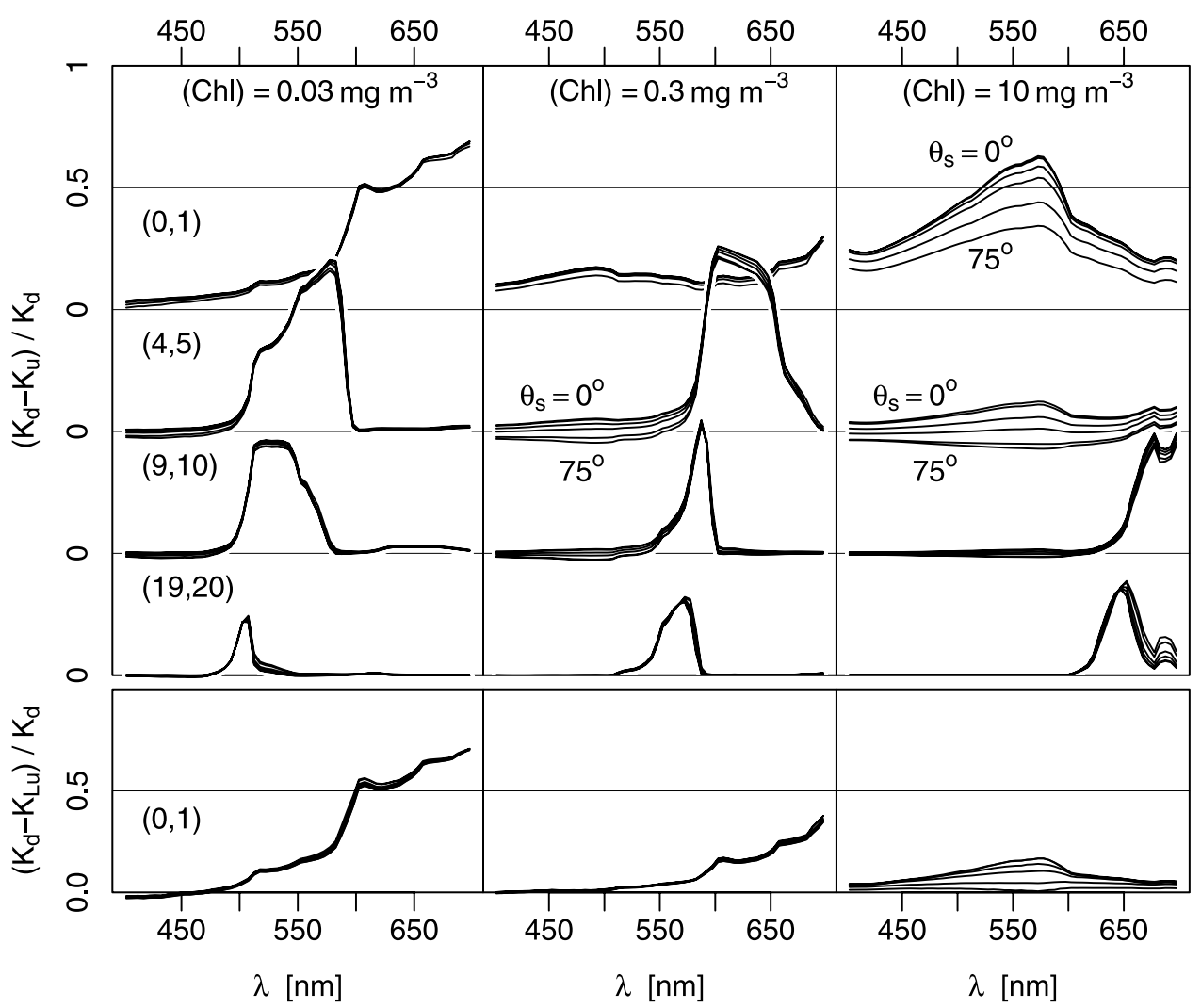

Figure 7. Spectral values of the ratio $\left(K_{d}-K_{u}\right) / K_{d}$, for three Chl values and different layers, namely the surface layer, a layer at mideuphotic depth, at $Z_{\text {eu }}$ and twice $Z_{\text {eu }}$ (from top to bottom); note that for clarity the curves corresponding to the different layers are shifted down by 0.5 . The lower row shows, for the same upper $(0,1)$ layer and the same Chl concentrations, the quantity $\left(K_{d}-K_{L u}\right) / K_{d}$, where $K_{L u}$ stands for the diffuse attenuation coefficient for the upward (nadir) radiance, $L u$.

[45] The present $R$ spectra differ slightly, at least in magnitude, from those derived from the previous approximate computations (as presented by MM01, their Figure 8, and here reproduced in Figure $8 \mathrm{~b}$ for visual comparison). There are three main, easily identified reasons for these differences. The first and most important one lies in the adoption for the approximate method (MM01) of a unique value (actually 0.33 ) for the $f$ factor. Such a constant value is a slight underestimate for low Chl but is overly low at high Chl, especially in the green $(\approx 570 \mathrm{~nm})$ part of the spectrum [see Morel et al., 2002, Figure 7]. Therefore the MM01 spectra are systematically, and in a varying manner, below those presently derived. The second reason is the ignorance in MM01 of the Raman effect, with the consequence that the present spectra that account for this effect are again above the previous ones. The relative enhancement occurs essentially in the red part of the spectrum and for low Chl $\left(\lambda>600 \mathrm{~nm}\right.$ and $\mathrm{Chl}<0.3 \mathrm{mg} \mathrm{m}^{-3}$; see below). The third reason, with more subtle consequences, apart from allowing the Sun angle dependence to be now put in evidence, lies in the use of an accurate method for solving the full radiative transfer problem.

[46] The comparison with the corresponding spectra computed in a purely elastic scattering mode quantitatively demonstrates that the radiant flux added by the Raman emission heightens $R$ in a significant way, by $5 \%$ (in the blue part of the spectrum) up to $15 \%$ (in the red part of the spectrum), when $\mathrm{Chl}=0.03 \mathrm{mg} \mathrm{m}^{-3}$ (see Figure 9a, which shows the ratio $R$ with $\operatorname{Raman} / R$ without Raman). This relative enhancement obviously diminishes when Chl (and hence elastic scattering by particles) increases; it remains below $1 \%$ when $\mathrm{Chl}=10 \mathrm{mg} \mathrm{m}^{-3}$. This increase depends slightly on the solar angle (it is maximum for $\theta_{s}=0$ ). Its spectral shape is directly governed by the spectral composition of the exciting (incident) radiation, with the appropriate wave number shift (about $-3350 \mathrm{~cm}^{-1}$ ).

[47] This representation could be fallacious inasmuch as the greatest relative increase occurs in the red part of the spectrum (at least for low Chl), where the reflectance itself is extremely low $(<0.1 \%$; Figure $8 \mathrm{a})$. Conversely, the minimal relative increase, in the blue part of the spectrum, affects the domain of high reflectance (about 10\% in blue waters). Therefore another representation is added (Figure 9b), which rests on the absolute difference $\Delta R(=R$ with Raman - $R$ without Raman) and shows the same results in a different way. The largest absolute increase in $R$ due to the Raman emission occurs in the blue part $(400-430 \mathrm{~nm})$ of the spectrum for low Chl or, although less marked, everywhere in the 400-570 $\mathrm{nm}$ band for moderate to high Chl. Numerical discrepancies between the present results and those of Haltrin and Kattawar 

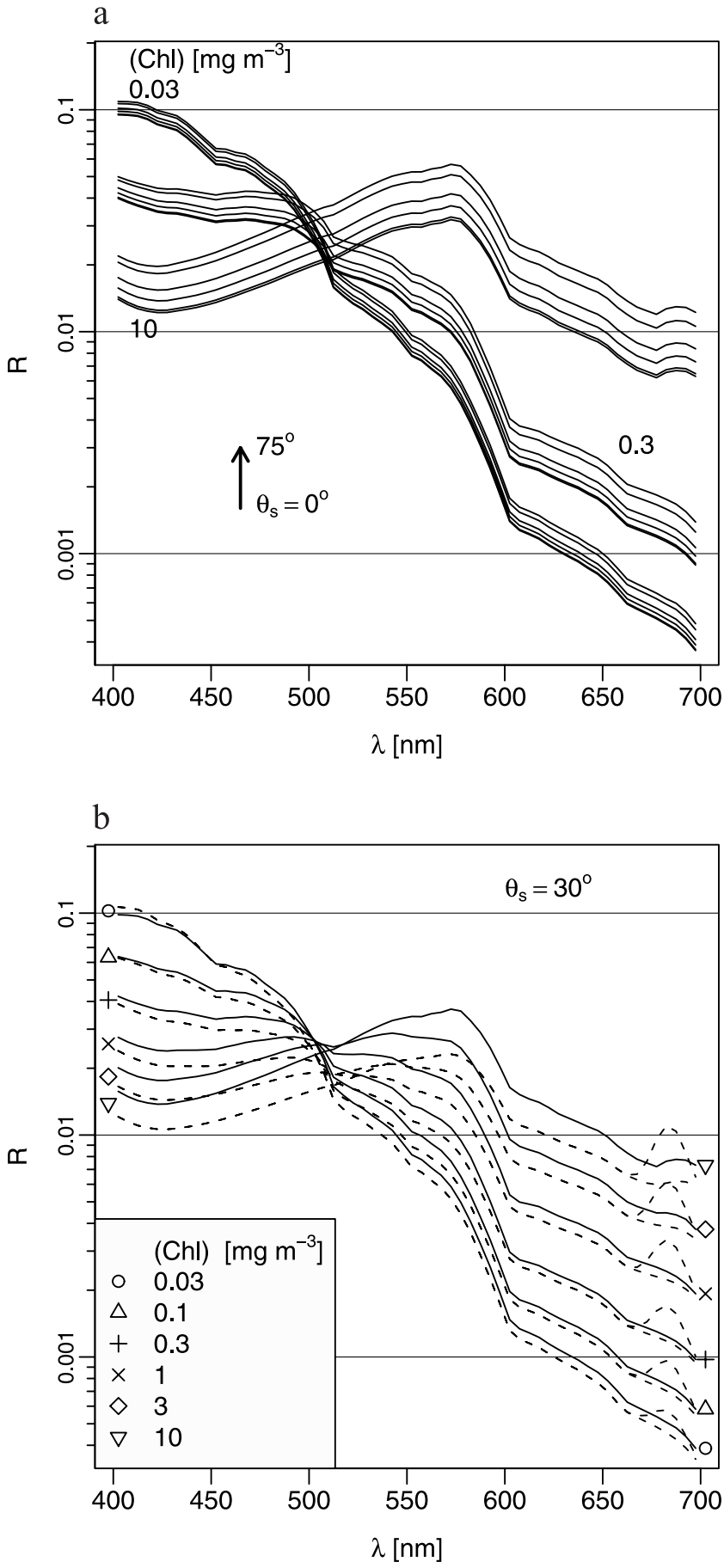

Figure 8. (a) Reflectance spectra at null depth for three selected Chl values and $\operatorname{six} \theta_{s}$ values; note that $R$ is steadily increasing with $\theta_{s}$. (b) Reflectance spectra at null depth and for a single $\theta_{s}$ value $\left(30^{\circ}\right)$ reproduced from Figure $8 \mathrm{a}$ (solid curves) but for all Chl values; the dashed reflectance spectra are reproduced from MM01, to which are added the Chl-a fluorescence peaks (see Appendix B).

[1991] originate from differences in the ways of modeling the IOPs and from their method of (approximately) solving the RTE; in addition, the rather high content in organic dissolved matter, covarying with $\mathrm{Chl}$ in their model, leads to a considerable fluorescence signal raising the reflec- tance. In contrast, the present results and those presented by Waters [1995] are very close.

\subsection{Reflectance at Increasing Depth Within the Water Column}

[48] Within a homogeneous water body, and inside the elastic scattering domain, the changes in $R$ with increasing depth remain limited. They result, as for all other AOPs, from the progressive rearrangement of the radiance field, from its initial structure imposed by the incident conditions of illumination within the upper layer, toward a steady (asymptotic) structure imposed by the medium itself. The exact relationship (derived from the definitions),

$$
R=\left(\bar{\mu}_{d}-\bar{\mu}\right) \bar{\mu}_{u} /\left(\bar{\mu}_{u}+\bar{\mu}\right) \bar{\mu}_{d},
$$

between $R$ and the three average cosines, in principle, allows the variations in $R$ to be understood; however, its interpretation is not intuitively easy. A simple guideline, however, can be found by observing that $R\left(0^{-}\right)$at the surface (actually, $f$ in equations (4a) and (4b)) is minimal when $\theta_{s}=0^{\circ}$ so that the light field rearrangement necessarily induces an increase in $R(z)$ with depth. The converse holds true when $\theta_{s}$ is large (here $\left.75^{\circ}\right)$ and $R\left(0^{-}\right)$is maximal; in this case, $R(z)$ will generally decrease with depth after some nonmonotonous variations near the surface due to the complex behavior of the $\bar{\mu}$ s (some instances are shown in Figure 10).

[49] The situation is quite different within the spectral domain, where inelastic scattering occurs and may become dominant. In the case of a completely isotropic radiance field, $\bar{\mu} \rightarrow 0$ and $\bar{\mu}_{d}$ and $\bar{\mu}_{u} \rightarrow 0.5$ so that $R$ tends toward unity. Actually, $R$ remains slightly $<1$ to the extent that the upper half-space, more lighted (by the exciting wavelengths) than the lower one, can reemit downward more Raman radiation than does the lower hemisphere in the upward direction [see Maritorena et al., 2000, equation (9)].

[50] Examples of the evolution with depth of the $R$ spectra are provided in Figure 11. In clear low-Chl waters the Raman emission (which already impacts the $R$ spectrum even at null depth; see above) leads to a drastic change in the red part of the spectrum at a depth which does not exceed one tenth of $Z_{\text {eu. }}$. At $Z_{\text {eu }}$ the Raman emission entirely governs the $R$ spectrum between 500 and $700 \mathrm{~nm}$, and $R$ is close to 1 . In contrast, for high Chl values the enhancement in the red part of the spectrum is limited, even at $Z_{\mathrm{eu}}$, but becomes noticeable at twice $Z_{\text {eu }}$. The increasing particle (elastic) scattering at high Chl (see Appendix A) partly explains the lower relative influence of the inelastic scattering; the second reason is directly related to the geometrical depth and to the absorption of the red radiation. Indeed, in reference to the two examples in Figure 11, the depth corresponding to $0.1 Z_{\text {eu }}$ is $17.8 \mathrm{~m}$ when $\mathrm{Chl}=0.03 \mathrm{mg} \mathrm{m}^{-3}$, whereas $Z_{\text {eu }}$ is at $12.5 \mathrm{~m}$ when $\mathrm{Chl}=10 \mathrm{mg} \mathrm{m}^{-3}$; to the extent that the absorption coefficient in the red part of the spectrum is essentially due to water and, as a consequence, is rather weakly depending on Chl (Appendix A), the number of red photons left nonabsorbed at $12.5 \mathrm{~m}$ is much larger than at $17.8 \mathrm{~m}$, by a factor of about 30 (or 10) at $\lambda=700$ (or $650 \mathrm{~nm}$ ).

\subsection{Scalar Irradiance and Downward Irradiance}

[51] At any depth the time rate of radiant energy locally absorbed per unit of volume $\left(\mathrm{W} \mathrm{m}^{-3}\right)$ is expressed as the 

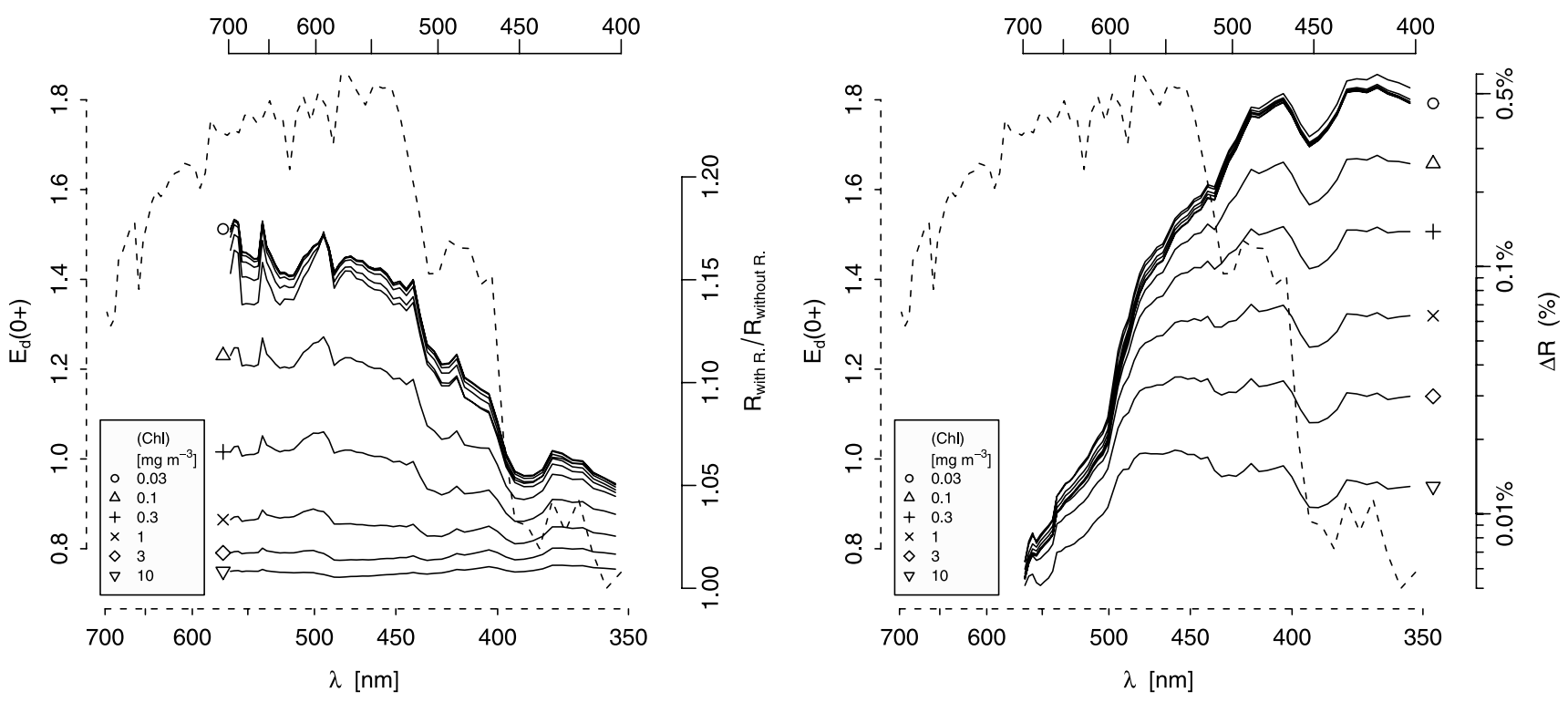

Figure 9. (a) Solid curves (right-hand scale, and top abcissae scale): spectral values of the ratio of reflectance including the Raman emission to reflectance when this effect is ignored (both at null depth). Dashed curve (left-hand scale, and bottom abcissae scale), spectral values of the downward irradiance above the surface (for $\theta_{s}=0$, and $\tau_{a}=0.2$ ). Actually, the abcissae scales are in wave number $(1 / \lambda$ ), even if for convenience the wavelengths are indicated, yet in the reverse direction; the upper scale is shifted with respect to the lower one (by $-3350 \mathrm{~cm}^{-1}$ in correspondence with the Raman shift). For Chl $=0.03 \mathrm{mg} \mathrm{m}^{-3}$ only, the various curves (from top to bottom) are for increasing $\theta_{s}$ values. (b) Absolute difference $\Delta R(=R$ with Raman $-R$ without Raman emission), plotted as in Figure 9a. The differences, $\Delta R$, as the reflectances themselves, are expressed as percent.

product of the scalar irradiance, $\stackrel{0}{E}$, and the local absorption coefficient,

$$
\Phi_{a}=\stackrel{0}{a} .
$$

When computing the heating rate, $a$ is the bulk absorption coefficient of the water body. When computing the energy directed toward the photosynthetic apparatus of algal cells, the relevant absorption must be the partial absorption coefficient due to the presence of phytoplankton. The absorbed power is obtained by integrating the product $a(\lambda) \stackrel{0}{E}(\lambda)$ over the appropriate wavelength domain. For a passive medium (no source) the quantity a $E_{0}$ can be rewritten as (an exact relationship)

$$
\stackrel{0}{E}=K_{d} E_{d}\left[1-R\left(K_{u} / K_{d}\right)\right] .
$$

$R$ is generally small (at least in case 1 waters), and the ratio $K_{u} / K_{d}$ most often is $<1$ (see above). Therefore neglecting the bracket leads to an overestimate of the product $(a E)$ by a few percents. This approximation has been used in previous studies [e.g., Morel, 1991; Morel and Antoine, 1994] when dealing with photosynthesis modeling or heating rate predictions. However, in these studies the $K_{d}$ variations with the solar angle were accounted for in a simplified manner (the so-called "geometrical" correction by Morel [1991]). The present computations provide an exact (and spectral) answer to this question, including when the Raman emission interferes (and when equation (7) does no longer hold true).

[52] From their definitions, it also follows that

$$
\stackrel{0}{E} / E_{d}=1 /(\bar{\mu}+\varepsilon),
$$

where $\varepsilon$, which represents the ratio $E_{u} / E^{0}$ (necessarily smaller than $R$ ), is a very small number in the spectral domain of purely elastic scattering. In this case, the $E / E_{d}$ ratio is approximately the reversed image of the $\bar{\mu}$ evolution (Figure 12). The maximal deviation (between $\stackrel{0}{E} / E_{d}$ and $\bar{\mu}^{-1}$ ) of about $-10 \%$ occurs in the blue-violet spectral domain and when Chl is $0.03 \mathrm{mg} \mathrm{m}^{-3}$, simply because $R$ (and $\varepsilon$ ) are maximal there. Within the upper layers the $\stackrel{0}{E} / E_{d}$ ratio steadily increases when $\theta_{s}$ increases, by about $30-45 \%$, when $\theta_{s}$ varies from 0 to $75^{\circ}$ (Figure 12 (top)). The spectral variations of the ${ }^{0} / E_{d}$ ratio are related to the single scattering albedo $(\varpi)$ that regulates the diffuse state established within the radiant field. For high $\varpi$ values (Appendix A) occurring at low $\mathrm{Chl}$ and short wavelengths, or at all wavelengths (particularly those around $560 \mathrm{~nm}$ ) for high $\mathrm{Chl}, \bar{\mu}$ is at a minimum; hence the ${ }^{0} / E_{d}$ ratios are maximal. This trend is reinforced for an increasing obliquity of the solar rays. The converse holds true when $\varpi$ is small, namely in the red part of the spectrum and for low Chl (Figure A1). In this case, $\bar{\mu}$ is maximal and ${ }^{0} / E_{d}$ minimal. With increasing depth (lower part of Figure 12), $E^{0} / E_{d}$ stabilizes around $\sim 1.5$ in the elastic domain, regardless of the Sun position.

[53] The situation is quite different when inelastic scattering comes into play and ultimately tends to dominate the light field. In such circumstances (and for appropriate wavelengths), $\bar{\mu}$ tends toward 0 , whereas $\varepsilon$ is no longer a small quantity because it tends toward $1 / 4$. Therefore the ratio ${ }_{E}^{0} / E_{d}$ tends toward 4 (Figure 12 (bottom)), which is obviously directly derivable for an isotropic radiant field. 


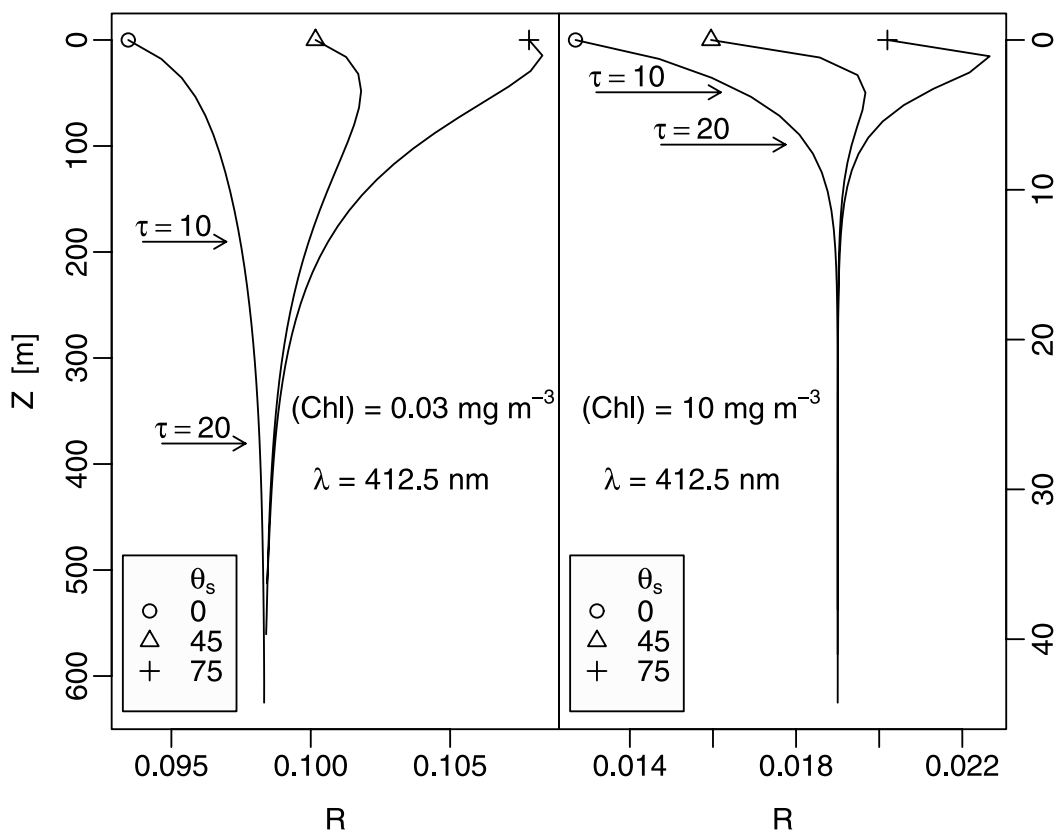

Figure 10. For three selected $\theta_{s}$ values and two Chl concentrations, as indicated, evolution of the reflectance at a specific wavelength with increasing geometric depth (in $\mathrm{m}$ ); the level corresponding to an optical depth $\tau=20$ is also displayed.

Note that the energy carried by this red radiation is minute compared to that transported by the elastically scattered radiation (but the isotropic character of this red light field is perhaps in keeping with camouflage strategies developed by some red colored organisms living at depth).

[54] For practical applications (photosynthesis studies) the spectrally integrated quantities over the PAR spectral domain are relevant, and Figure 13 shows some selected examples of the ratio ${ }_{E}(\mathrm{PAR}) / E_{d}(\mathrm{PAR})$. Even if, as seen before, the geometric change in $Z_{\mathrm{eu}}$ is minute when $\frac{0}{E}$ replaces $E_{d}$ in its definition, it is worth noting that the energy really available at all levels within the euphotic zone, which is correctly expressed by $E$, is always largely above that one which is inferred from $E_{d}$. In addition, the excess of $E$ compared to $E_{d}$ is heavily depending on the solar position particularly for the upper layers. Only at depths of about $2 Z_{\text {eu }}$ does the ${ }_{E}$ (PAR) $/ E_{d}(\mathrm{PAR})$ ratio converge

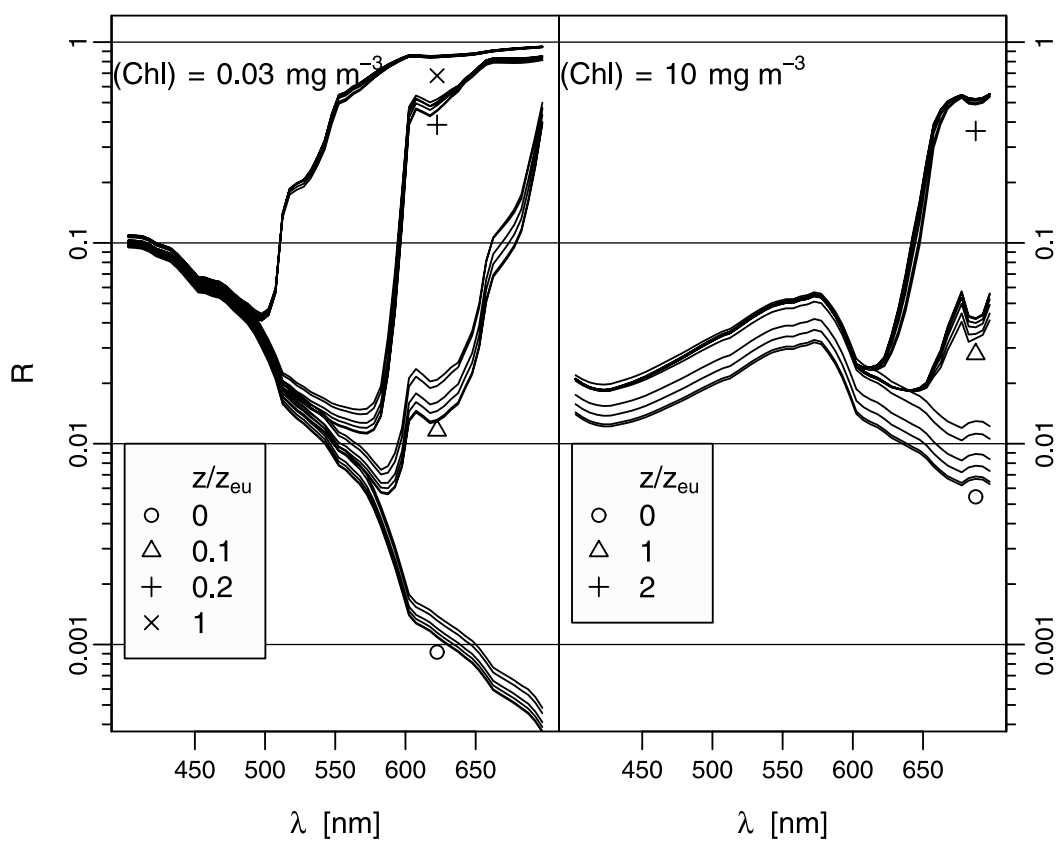

Figure 11. Evolution with depth of the reflectance spectra, for two selected chlorophyll concentrations (left- and right-hand panels); the reference depths are the surface $\left(0^{-}\right)$, and the depths corresponding to $1 / 10,2 / 10 Z_{\mathrm{eu}}$, to $Z_{\mathrm{eu}}$, and twice $Z_{\mathrm{eu}}$, as indicated. 


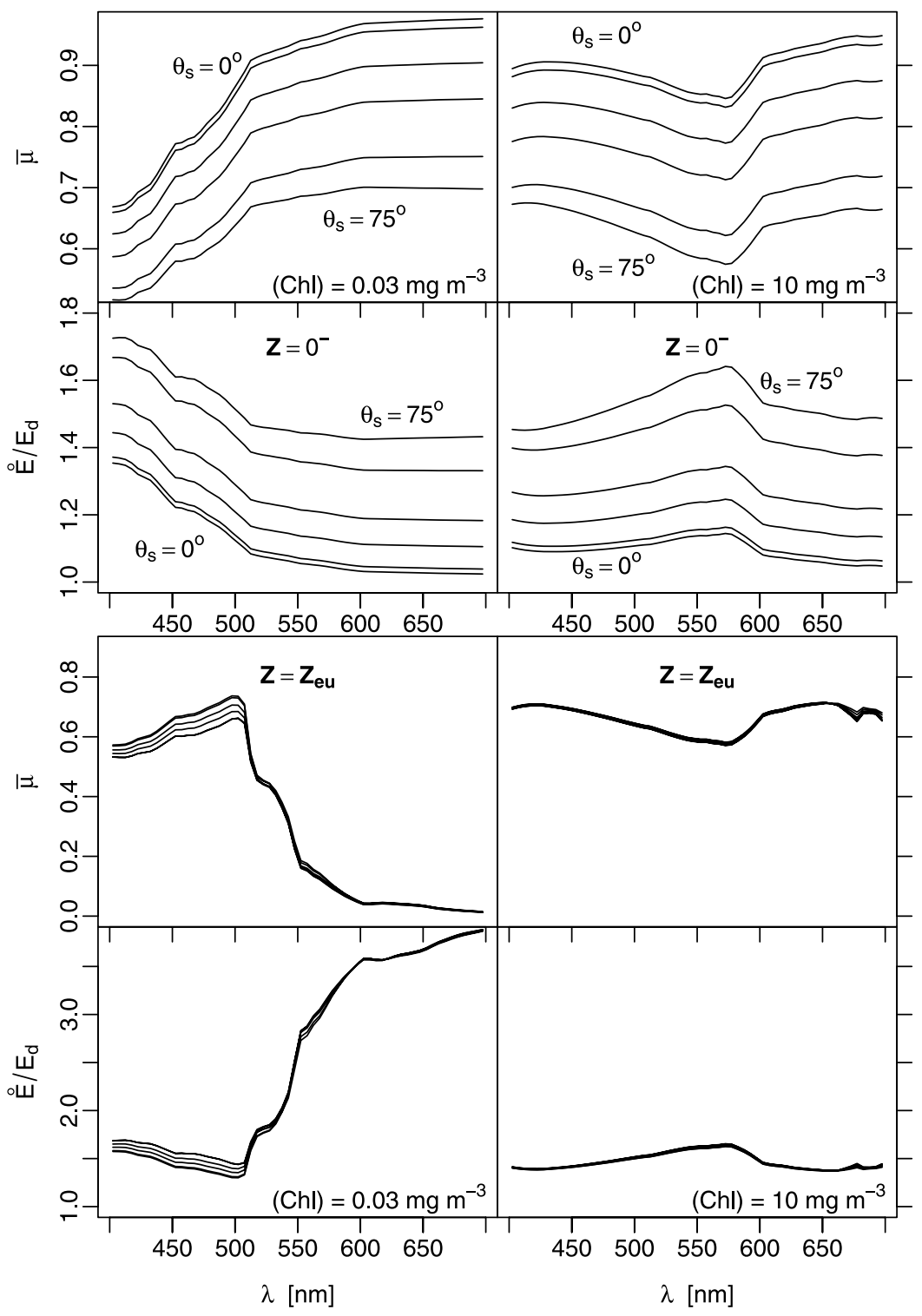

Figure 12. Spectral distribution of the quantities $\mu$ and $0 E / E_{d}$. The four upper panels are for the depth $0^{-}$(just beneath the surface), the four lower panels for $Z=Z_{\mathrm{eu}}$. The left-hand column is for $\mathrm{Chl}=0.03 \mathrm{mg}$ $\mathrm{m}^{-3}$, and the right-hand one is for $\mathrm{Chl}=10 \mathrm{mg} \mathrm{m}^{-3}$.

around a limiting value (from 1.45 to 1.62 ; Figure 13). This effect was accounted for in a previous study [Morel, 1991], yet in an approximate manner. It consisted of making use of a result obtained by Kirk [1984] that provides at ${ }_{0}$ each wavelength $\lambda$ an average value of the ratio $K_{d} / a\left(\cong E / E_{d}\right)$, valid for the entire euphotic zone and depending on the Sun position through $\bar{\mu}_{d, w}$. This approximate approach can be considerably improved by using the present results.

\subsection{Average Cosines}

[55] The average cosine for downward irradiance beneath the surface $\left(\bar{\mu}_{d, w}\right)$ is involved in several computations and approximations, as previously used, for instance, when dealing with $K_{d}$ (Figure 4). In such approximations the average cosine $\bar{\mu}_{d, w}$ is generally predicted from simple geometric considerations (Snell's law applied to $\theta_{s}$ ) and by accounting for varying contributions of direct sunlight and diffuse sky light. The upward radiances redirected downward by total internal reflection, which also impacts (decreases) the $\bar{\mu}_{d, w}$ value [Gordon et al., 1975], is most often ignored. This question can be briefly examined with the data in hand and by considering typical (extreme) cases (Figure 14).

[56] Four spectra of $\bar{\mu}_{d}\left(0^{+}\right)$(the $\bar{\mu}_{d}$ value in air, above the surface) are shown for a Sun-zenith angle $\theta_{s}=0^{\circ}$ and when $\tau_{a}=0.2$ or 1 , or with $\tau_{a}=1$ only when $\theta_{s}=60^{\circ}$, and finally for an overcast sky. The more important contribution of the sky light in the blue part of the spectrum explains the slight decrease in $\bar{\mu}_{d}\left(0^{+}\right)$and the distinct regular curvature. This convexity tends to vanish for higher $\theta_{s}$ values [see also Morel, 1991, Figure 3; Sathyendranath and Platt, 1997, Figure 7]. Just below the surface, the associated $\bar{\mu}_{d}\left(0^{-}\right)$ spectra are obviously well above those of $\bar{\mu}_{d}\left(0^{+}\right)$, as a result of the increased verticality of the downward stream following refraction. The spectral shape is also slightly modified because about half of the upward irradiance is reflected 


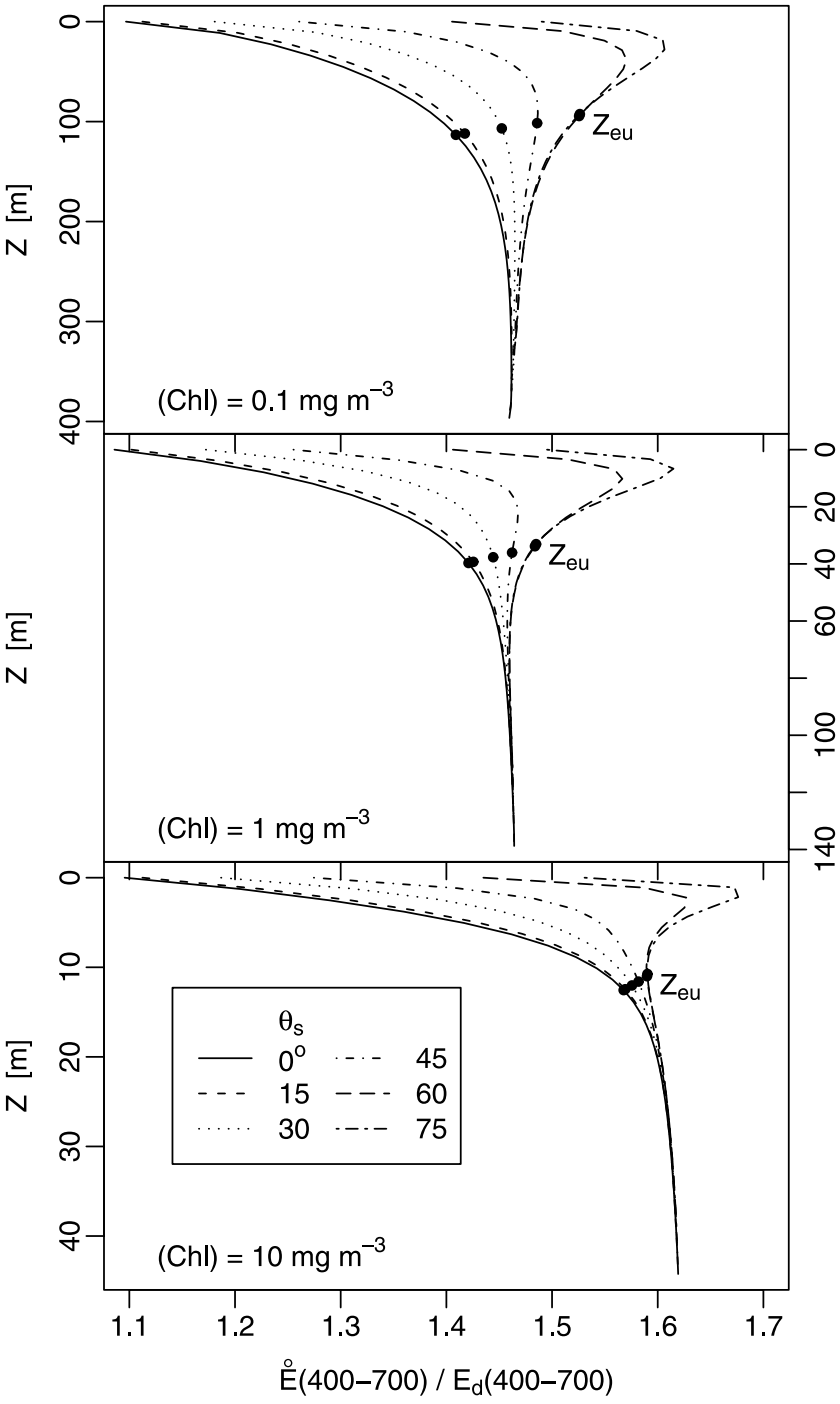

Figure 13. Variations with solar zenith angle $\left(\theta_{s}\right)$ and depth $(Z)$ of the ratio of the scalar irradiance to the downward irradiance computed for the entire photosynthetic domain (400-700 nm). The three panels correspond to three $\mathrm{Chl}$ values as indicated. In each panel are also plotted the depths corresponding to the depth of the euphotic layer (which varies with $\theta_{s}$ ).

down (with slant directions) causing a decrease in $\bar{\mu}_{d, w}$, particularly at those wavelengths which correspond to high reflectance values. When $\mathrm{Chl}=0.03 \mathrm{mg} \mathrm{m}^{-3}$, this effect is clearly seen in the blue-violet part of the spectrum, whereas when $\mathrm{Chl}=10 \mathrm{mg} \mathrm{m}^{-3}$, the depression in $\bar{\mu}_{d}\left(0^{-}\right)$occurs around $560 \mathrm{~nm}$, in both cases when $R$ is a maximum.

[57] The evolution of $\bar{\mu}_{d}$ along with depth is easy to interpret; selected instances are displayed in Figure 15. Considering at first the situation with $\mathrm{Chl}=0.03 \mathrm{mg} \mathrm{m}^{-3}$ and the spectral domain $(\lambda<500 \mathrm{~nm})$, where the Raman emission does not significantly interfere, the dependence on solar angle progressively diminishes with increasing depth, but is still obviously present at the depth corresponding to $Z_{\text {eu }}(178.6 \mathrm{~m})$. Actually, at this geometrical depth, the optical depth $\tau(=c z)$ is 9.98 or 9.57 for $\lambda=400$ or $500 \mathrm{~nm}$. If $\mathrm{Chl}=10 \mathrm{mg} \mathrm{m}^{-3}$ (leading to $Z_{\mathrm{eu}}=12.55$ ), $\tau$ is between 34 and 38 everywhere within the visible spectrum, which explains that the sensitivity to the Sun position has practically disappeared (the asymptotic regime is not far from being "reached", see Appendix C). An intermediate behavior is noticed for $\mathrm{Chl}=1 \mathrm{mg} \mathrm{m}^{-3}$.

[58] In the spectral domain where the Raman emission occurs, the quasi-isotropy of the light field generated by this process leads to $\bar{\mu}_{d}$ tending toward 0.5 . This is clearly seen for $\lambda>600 \mathrm{~nm}$ when $\mathrm{Chl}=0.03 \mathrm{mg} \mathrm{m}^{-3}$ (even at mideuphotic depth); the same trend is detectable at $Z_{\text {eu }}$ for longer wavelengths (approaching $700 \mathrm{~nm}$ ) when $\mathrm{Chl}=1 \mathrm{mg}$ $\mathrm{m}^{-3}$ and remains absent for $\mathrm{Chl}=10 \mathrm{mg} \mathrm{m}^{-3}$ (in this case, the effect begins to appear near $700 \mathrm{~nm}$ at 1.5 or $2 Z_{\mathrm{eu}}$ ).

[59] As far as the average cosines for upward irradiance, $\bar{\mu}_{u}$, are concerned, the amplitudes of their variations are rather restricted (Figure 15). They are confined within the range $0.35-0.45$ in the elastic domain, and less dependent on the solar position than were the $\bar{\mu}_{d}$. For the same reason as above, when the light field becomes dominated by the Raman emission $\mu_{u}$ increases and also tends toward, or even slightly exceeds, 0.5 .

[60] The average cosine for the entire radiant field $(\bar{\mu})$ has been previously examined in relation with the behavior of the

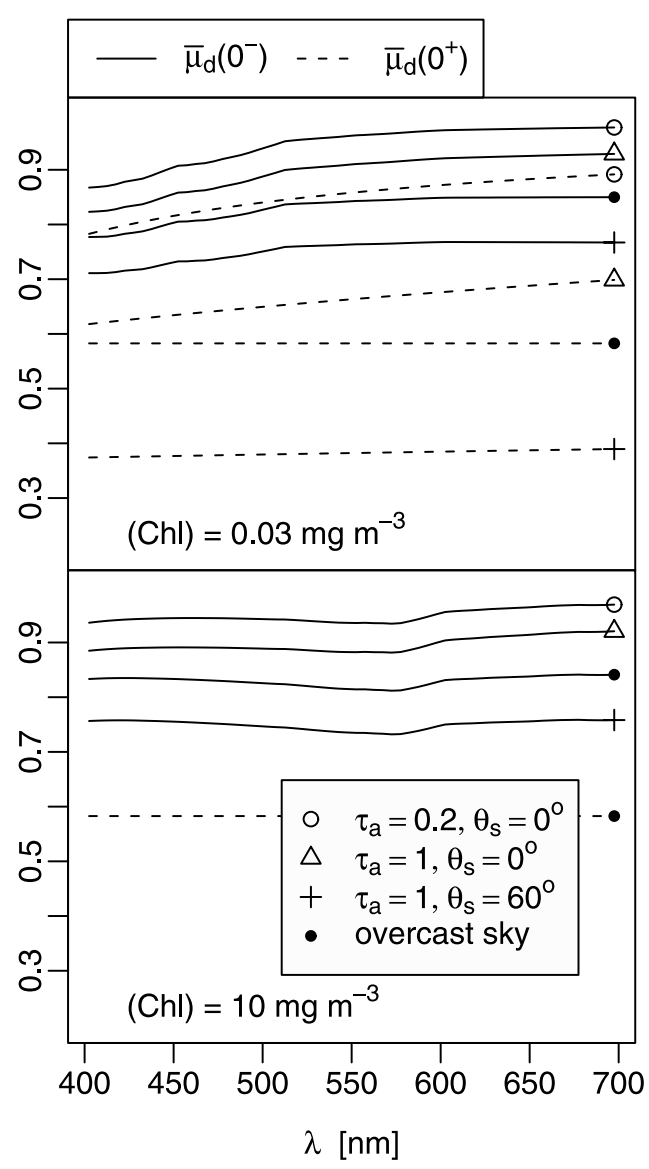

Figure 14. Spectral values of the average cosine for downward irradiance above the surface, $\bar{\mu}_{d}\left(0^{+}\right)$, and below the surface $\bar{\mu}_{d}\left(0^{-}\right)$, for various Sun-zenith angles and aerosol optical thickness, and for an overcast sky, as indicated. Upper and lower panels are for the extreme values of the chlorophyll range. 


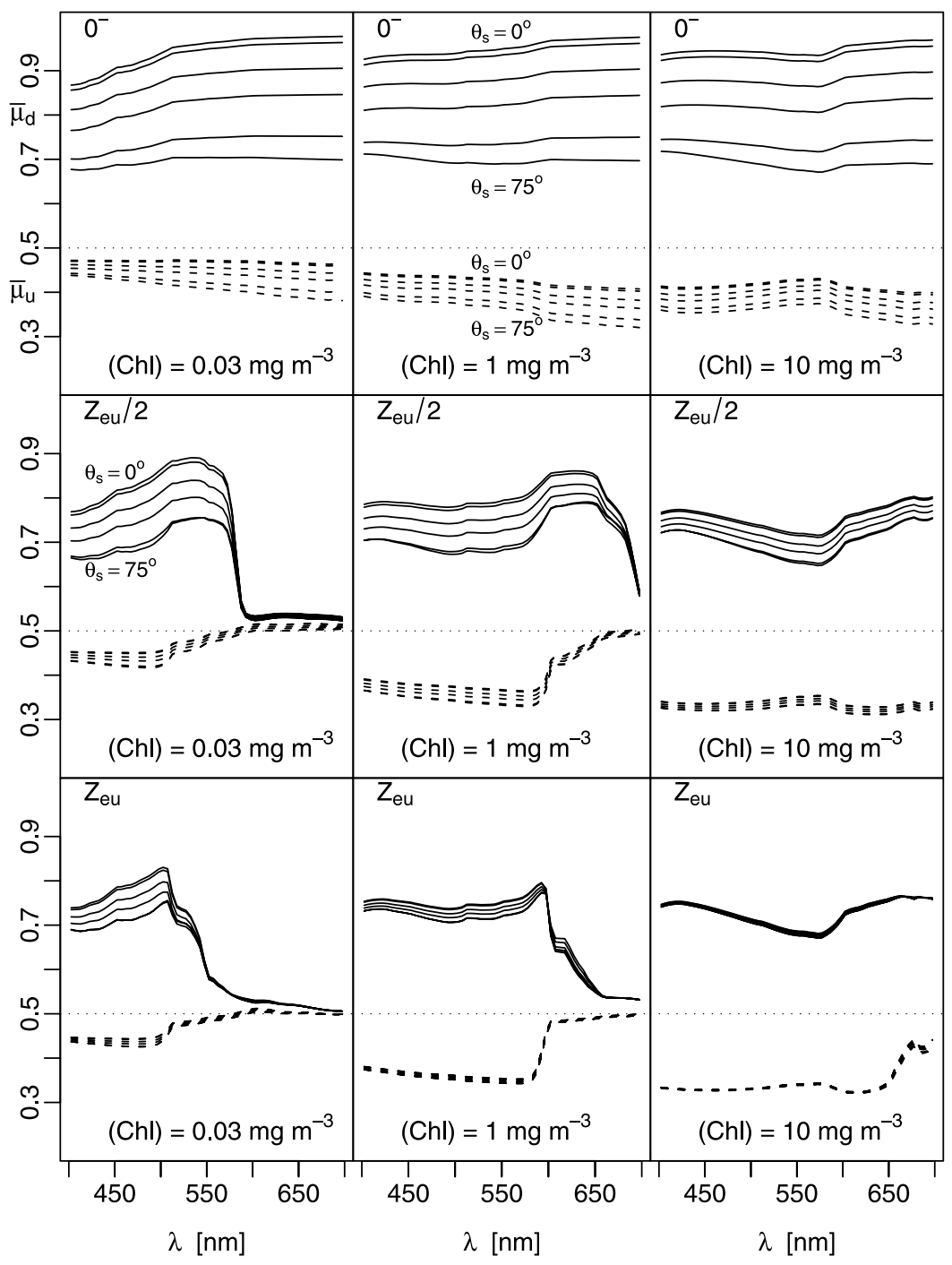

Figure 15. Spectral distribution of the average cosines, for downward irradiance, $\bar{\mu}_{d}$ (solid lines) and for upward irradiance, $\bar{\mu}_{u}$ (dashed lines). From top to bottom, three depths are considered, $0^{-}, Z_{\mathrm{eu}} / 2$, and $Z_{\mathrm{eu}}$. The three columns are for three Chl values, as indicated.

$\stackrel{0}{E} / E_{d}$ ratio. The exact geometrical relationship (equation (5)), which relates the 3 cosines $\left(\bar{\mu}, \bar{\mu}_{d}\right.$, and $\left.\bar{\mu}_{u}\right)$ and $R$ can be used for numerical check but is rather unpractical when trying to figure out the associated variations.

\subsection{Asymptotic (or Diffuse) Regime}

[61] The asymptotic values of the AOPs are uniquely determined by the IOPs of the medium and are approached when the depth increases sufficiently, and provided that the medium remains optically homogeneous [Preisendorfer, 1961]. They are independent from the way the body is illuminated from above, and actually become other IOPs (related to the basic IOPs, in a complex, nonanalytic way). In the previous figures several AOPs have been presented as a function of depth within homogeneous water bodies. Their convergence at sufficient depth toward a unique value whatever the incident Sun angle, denotes that an asymptotic diffuse regime is setting up. Is the asymptotic regime "reached" at the depth of the euphotic layer (in $Z_{\mathrm{eu}}$ ) is a practical question which deserves examination.
[62] Globally, i.e., for the entire (400-700) spectrum, Figure 13 shows that the situations differ according to the chlorophyll concentration: when $\mathrm{Chl}=10 \mathrm{mg} \mathrm{m}^{-3}$, independence with respect to the incident irradiation is not far from being reached at $Z_{\text {eu }}$, while the same approximate convergence would not be observed before $2 Z_{\text {eu }}$, when $\mathrm{Chl}=0.1 \mathrm{mg} \mathrm{m}^{-3}$. By considering another sensitive AOP, namely the spectral $\bar{\mu}_{d}$ values in $Z_{\text {eu }}$ (Figure 15), the same conclusions can be drawn, and for all wavelengths, when $\mathrm{Chl}=10 \mathrm{mg} \mathrm{m}^{-3}$. In contrast, when $\mathrm{Chl}=0.03 \mathrm{mg} \mathrm{m}^{-3}$, the $\bar{\mu}_{d}$ values are still Sun angle-dependent when $\lambda<550 \mathrm{~nm}$, which confirms that for the penetrating radiation the diffuse regime is not established. For $\lambda>550 \mathrm{~nm}$, i.e., this spectral domain where the transmitted radiation is replaced by the internal Raman source, the usual concept of asymptotic diffuse regime (resulting from the progressive rearrangement of the elastically scattered radiation) is replaced by that of a radiative regime set up within a self-illuminating medium, and thus characterized by a quasi-isotropic radiant field, with both $\bar{\mu}_{d}$ and $\bar{\mu}_{u}$ converging toward 0.5 . 
[63] The differences noted above are not surprising. Indeed, the geometrical depth $Z_{\text {eu }}$, which is relevant for photosynthesis studies, has no particular significance in the radiation transport; the appropriate (dimensionless) quantity is the optical depth $\tau(=c Z)$. Graphs of the $\tau(\lambda)$ values at the level of $Z_{\text {eu }}$ which are displayed in Appendix A (Figure A2), show that, whereas the $\tau(\lambda)$ values are all above 35 , when $\mathrm{Chl}=10 \mathrm{mg} \mathrm{m}^{-3}$, they are only about 10 in the blue-green part of the spectrum, when $\mathrm{Chl}=0.03 \mathrm{mg} \mathrm{m}^{-3}$. Therefore in the first case the diffuse regime is more closely approached than in the second case. The rate of approach actually is essentially regulated by the fraction, $\tau_{b}$, of $\tau$, which corresponds to scattering (with $\tau_{b}=b Z=\varpi \tau$ ), because scattering is needed to rearrange the light field. In contrast, the complementary fraction, $\tau_{a}(=a Z=(1-\varpi) \tau)$, fixes the rate of photons disappearance without affecting their angular distribution (see also a more general discussion in Appendix C).

\section{Discussion and Applications}

[64] All the results presented above are rigorously derived via "exact" radiative transfer computations [Mobley et al., 1993]; it cannot be claimed, however, that they are exact in the sense that they rest on input parameters, namely the IOPs of case 1 waters, which inevitably are mean values. These values are believed to be the most representative on average of the natural conditions, but are only mean values. Therefore predictions from, or validation of, the theoretical results versus actual AOP measurements may show discrepancies, the origin of which is to be found in differences between the adopted mean IOPs and those naturally existing. Nonetheless the trends in all AOPs parameters and quantities are certainly well captured.

\subsection{Euphotic Layer Depth}

[65] As seen above, the various definitions of the euphotic layer have no practical impact on the resulting depth. In contrast, the dependence upon the solar angle is in no way a negligible phenomenon, particularly in clear low-Chl waters. Time- or Sun-independent predictions (as by Morel [1988]) could advantageously be tuned when dealing with high latitudes and permanent low solar elevation. And for primary production modeling, this dependence (or as a consequence, the variation of $K_{\mathrm{PAR}}$ with $\theta_{s}$ ) which is generally ignored, might easily be accounted for. A "correction" following the variations in the quantity $\left(1-\cos \theta_{\mathrm{sw}}\right)$ is definitely an overcorrection (even for extremely clear skies and low-Chl waters), and the use of a linear formula as (1 $\alpha \cos \theta_{\text {sw }}$ ), with $\alpha \cong 0.4$, could be a rough approximation, if the resort to a more precise correction, based on lookup tables is not envisaged.

\section{2. $K$ Coefficients}

[66] The approximate Gordon's formula (equation (2)) which implies a rather wide variation of the near-surface $K_{d}$ can be safely used, except in the red part of the spectrum (beyond $600 \mathrm{~nm}$ ) for low-Chl $\left(<0.3 \mathrm{mg} \mathrm{m}^{-3}\right)$ waters, because of the Raman effect. To the extent that the absorption coefficient is now routinely determined at sea (together with $K_{d}$ ), and provided that the accuracy of these determinations is sufficient, this formula could be used for a closure, allowing measurements of $b_{b}$ to be confirmed, or the $b_{b}$ value to be predicted if not determined.
[67] Extrapolation of radiometric quantities (such as upward and downward irradiances, or nadir radiance) toward the surface (at $0^{-}$) is a recurrent need, especially for obtaining "sea truth" data (in the perspective of ocean color remote sensing validation). In the extrapolation process, a mutual help of the $K_{d}, K_{u}$, and $K$ coefficient for nadir radiance $\left(K_{L u}\right)$ could be expected from their relative closeness, if any. Unfortunately, this is not the case (even if the Raman domain is excluded). Indeed, the numerical relationships between these coefficients are not particularly simple or easy to handle (Figure 7, layer 0,1 ). The $K_{L u}$ coefficients are similar to, but not coincident with, the $K_{u}$ coefficients (actually they are less Sun angle-dependent at high Chl), so that their relative differences with $K_{d}$ are not the same (compare the uppermost panels, and lower panels in Figure 7). The resort to available lookup tables nevertheless remains a possibility, which would allow a quality control of the extrapolations which have been made by checking the internal consistency of the various $K$ coefficients.

\subsection{Reflectance}

[68] The variations with $\theta_{s}$ of spectral reflectances just beneath the surface (or in other words of the $f$ factor; equations (4a) and (4b) are considerable (a possible doubling); they have already been examined together with the bidirectional effects [Morel et al., 2002]. Two consequences of these Sun-induced variations deserve some additional comments in relation to the remote sensing of ocean color. In principle, and under the proviso that the atmospheric correction is accurately performed, a single spectral reflectance (or equivalently any specific normalized water-leaving radiance) at a given wavelength can be used as a piece of information when interpreting ocean color data. This is the case, for instance, in view of detecting turbid case 2 waters through their "anomalously high" reflectances in the green part of the spectrum [Bricaud and Morel, 1987]. To be efficient and accurate, the detection threshold must be made varying with the solar elevation (although it was kept constant in the above reference, as this effect was not known). The Sun's position is also to be accounted for in the perspective of calibrating ocean color sensors via radiometric measurements at sea [Morel and Mueller, 2002].

[69] Most of the algorithms make use of one or several ratios of reflectances at two wavelengths; the influence of the Sun position is greatly reduced for such ratios, although it is not absent as the slopes $\left(S_{f}\right.$ in equation (4)) slightly differ according to the wavelength. If ratios of reflectances at two wavelengths, such as $R(445) / R(555)$ and $R(490) / R(555)$, shown in MM01 (their Figure 11), are separately plotted for each of the various $\theta_{s}$ values, the curves (as a function of Chl) remain very close; at least, the distance between them is less than the distance between actual data and the mean curve. Therefore within the accuracy of such algorithms, this effect can be neglected, as far as the irradiance reflectance ratio are considered; this conclusion is to be nuanced if bidirectional reflectances are considered.

[70] The effect of Raman scattering, which has been accurately quantified, has a minute impact on the shape and magnitude on the reflectance spectra at null depth, and thus on algorithms as above mentioned. The situation is completely different at depth (and in the red part of the 


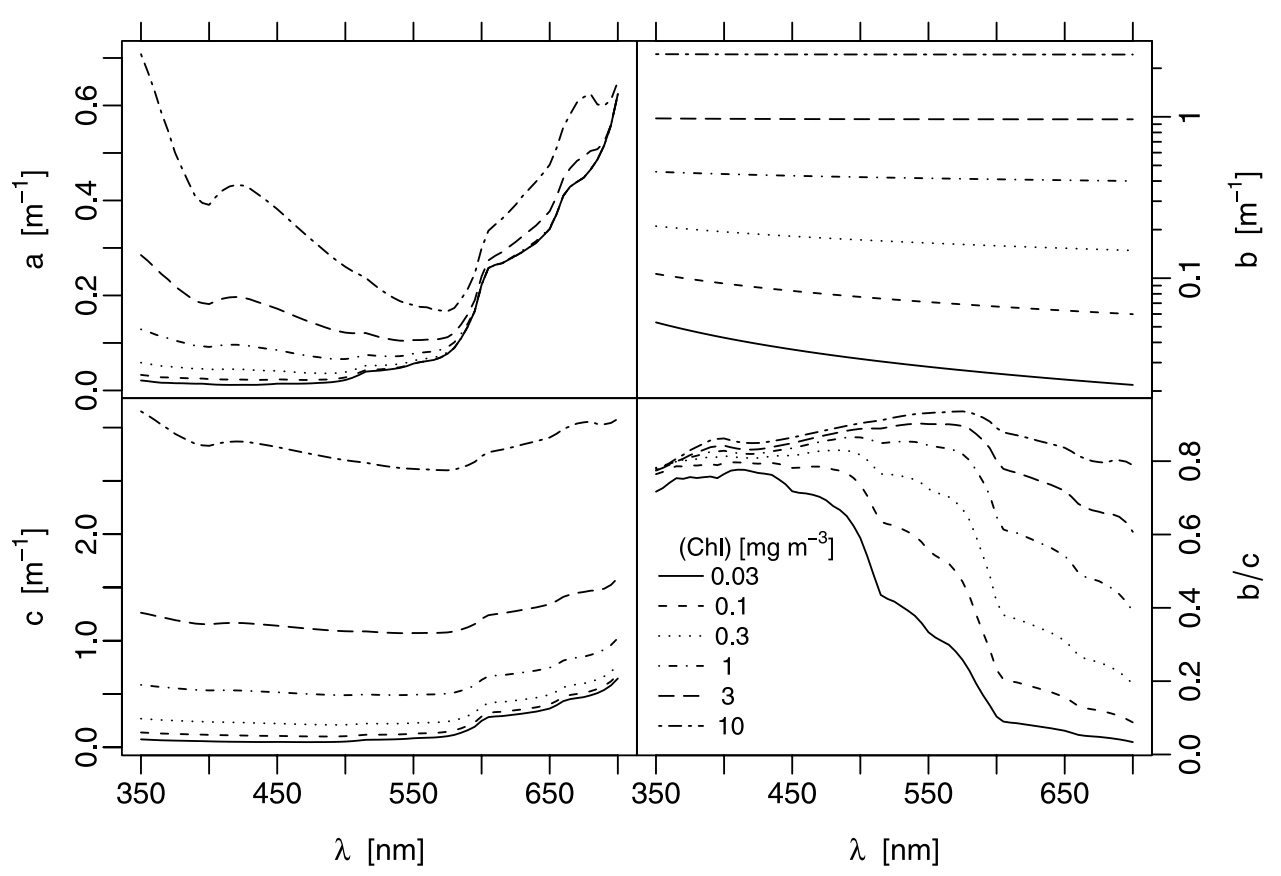

Figure A1. Spectral values of the IOPs ( $a, b, c$, absorption, scattering, and attenuation coefficients) used as inputs for the RTE computations for case 1 waters and for discrete Chl values, as indicated. In addition, the corresponding spectra of the single scattering albedo $(\varpi=b / c)$ are shown.

spectrum) as already accounted for when studying the chlorophyll fluorescence yield [Maritorena et al., 2000].

\subsection{Scalar Irradiance Verus Downward Irradiance}

[71] An accurate estimate of the scalar irradiance $E$ is particularly needed for primary production studies (field experiment or modeling). The obvious answer is just to make the appropriate measurement and develop a parameterization of this radiometric quantity as well as of the diffuse attenuation coefficients, $K_{0}\left(\lambda, \mathrm{Chl}, \theta_{s}\right)$, related to this irradiance. To the extent that such measurements are by far less common than those of the downward irradiance, and, as a consequence, the databank allowing a parameterization to be developed is relatively poor, the indirect answer which consists of transforming $E_{d}$ into $\stackrel{0}{E}$ remains operative. The spectral domain where inelastic scattering prevails, and the $\underset{E}{E} / E_{d}$ ratio takes exceptional values (up to 4 ), can be disregarded, as the energy transported via the Raman emission is extremely low. For the spectral domain corresponding to the elastically transmitted radiation, this ratio is highly dependent on the solar position within the upper layers, and meanwhile it tends, at depth, to stabilize around a value as high as 1.5. A comparison with the geometric correction used by Morel [1991] for deriving this ratio shows that his approximation has led to a global underestimate. The consequences of this underestimate remain to be explored, by keeping in mind that the photophysiological parameters, also involved in the photosynthesis model, will never be known with the accuracy of physical parameters.

\section{Appendix A: Inherent Optical Properties Used as Inputs for the RTE}

[72] The inherent optical properties of case 1 waters with varying chlorophyll concentration, which are used as inputs in the radiative transfer computations, are graphically displayed (Figure A1), together with some other derived quantities. The absorption, $a(\lambda)$, scattering, $b(\lambda)$, and their sum, the attenuation coefficient $c(\lambda)$ form the basic set. Absorption values beyond $620 \mathrm{~nm}$ and when $\mathrm{Chl}<0.3 \mathrm{mg}$ $\mathrm{m}^{-3}$, when derived from the iterative process (see text), may be slightly (erratically) below those for pure water, because of numerical instability. They are simply reset to those of pure water, which entails a negligible inaccuracy (actually, the negative differences are smaller than the uncertainty attached to the pure water values). The scattering coefficient, $b(\lambda)$, is spectrally neutral when $\mathrm{Chl}=10 \mathrm{mg} \mathrm{m}^{-3}$, and becomes progressively wavelength-dependent when $\mathrm{Chl}$ decreases, for two reasons; firstly the influence of the molecular scattering (with a $\lambda^{-4.3}$ dependency) is regularly increasing, and secondly, the particle scattering is itself made progressively wavelength-dependent (according to a varying negative exponent (see text)).

[73] The attenuation coefficient spectra span about two orders of magnitude for the chlorophyll range considered; they are obviously flatter than the absorption spectra, with a minimum which migrates from $435-440 \mathrm{~nm}$ when $\mathrm{Chl}=$ $0.03 \mathrm{mg} \mathrm{m}^{-3}$, to $565 \mathrm{~nm}$ when $\mathrm{Chl}=10 \mathrm{mg} \mathrm{m}^{-3}$.

[74] The single scattering albedo, $\varpi$, is a crucial (dimensionless) parameter which governs the nature of the radiation transfer within the water body, either dominated by absorption or by scattering ( $\varpi$ low or high, respectively). The $\varpi$ spectra show that in case 1 waters both these situations occur in the red part of the spectrum, according to the Chl value. In contrast, in the near UV and blue part of the spectrum $(\lambda<480 \mathrm{~nm})$, all the $\varpi$ values are close to 0.8 , whatever Chl.

[75] The ratio of molecular scattering to total (molecular plus particle) scattering, $\eta$, is also an important parameter as it governs in great extent the shape of the global volume 


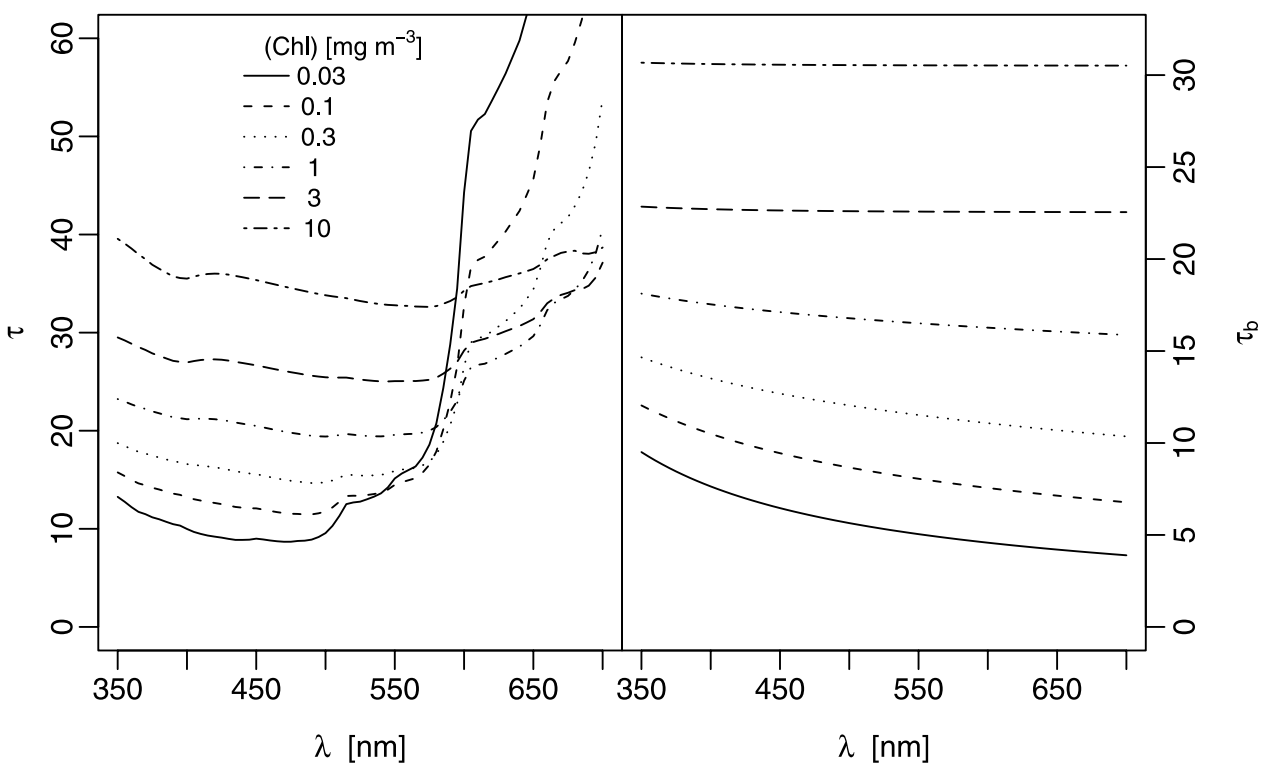

Figure A2. For discrete Chl concentrations, spectral values of the optical depth $(\tau)$ and scattering optical depth $\left(\tau_{b}\right)$ in $Z_{\text {eu }}$, i.e., the geometrical depth of the euphotic zone (when the Sun-zenith distance is zero).

scattering function. The particle scattering phase function itself is obtained as a weighted sum of two functions for small-sized and large-sized particle populations; the weighting coefficients depend on Chl, so that the shape of the resulting global phase function (as well as the backscattering probability) are progressively changing with the chlorophyll concentration (see the discussion and Figure 3 in the work of Morel et al. [2002], not reproduced here).

[76] Finally, a graph (Figure A2) shows the spectral variations of the particular $\tau(\lambda)$ values computed for the depth of the euphotic layer by using the $Z_{\text {eu }}$ values in the first line of Table 2, and for the various Chl values; are also shown the spectral values of the scattering optical depths, $\tau_{b}(\lambda)$ at the same $Z_{\text {eu }}$ level.

\section{Appendix B: Chl-a Fluorescence by Phytoplanktonic Algae}

[77] The way of accounting for fluorescence in the radiative transport computations is the same as for the Raman scattering. As Chl-a fluorescence is partly a physiological response of the photosynthetic apparatus of algae, and not a purely physical phenomenon (as Raman emission, or as well fluorescence emission by isolated Chl-a molecules in a solvent), the parameters regulating this process are less stable and predictable. All wavelengths are involved in the excitation, provided that the corresponding photons can be absorbed by phytoplankton. Therefore a simulation implies that the spectral distributions of both the available (scalar) irradiance, and of the algal (species-dependent) absorption are locally known. In contrast to Raman emission, the fluorescence emission band is spectrally fixed; it is centered on $685 \mathrm{~nm}$, and approximately exhibits a Gaussian shape with a half-band width of about $20 \mathrm{~nm}$. The quantum yield $\left(\phi_{f}=\right.$ number of photons emitted per number of absorbed photons) is a photophysiological and ecological quantity, that is able to vary by at least a factor 10 (likely between $5 \times 10^{-3}$ and $5 \times 10^{-2}$ ). It is generally minimal near the surface (and at noon), and increasing with diminishing light, i.e., with increasing depth and (or) solar angle [Maritorena et al., 2000; Morrison, 2003]. Because of the localized and time-dependent character of these parameters, generic computations using fixed values are rather pointless or academic. Specific computations, however, are obviously possible when the appropriate parameters are known.

[78] In Figure 8b, are shown Chl-a fluorescence peaks added to the reflectance spectra just beneath the surface. They were produced with the following input parameters and assumptions. The incident irradiation above the surface, $E_{d}\left(0^{+}, \lambda\right)$, is that of a clear sky $\left(\tau_{\text {aer }}=0.2\right)$ with $\theta_{s}=30^{\circ}$. The scalar irradiance $E^{0}\left(0^{-}, \lambda\right)$ below the interface, expressed in terms of photons (per second) is determined and combined with the absorption coefficient of phytoplankton, $a_{\phi}(\lambda)$, to compute at a given level the power absorbed by algae, $\Phi_{a}$, (photons $\mathrm{s}^{-1} \mathrm{~m}^{-3}$ ) through

$$
\Phi_{a}=\int_{400}^{660} a_{\phi}(\lambda) \stackrel{0}{E}\left(0^{-}, \lambda\right) d \lambda,
$$

where the spectral limits are those of the excitation spectrum; $a_{\phi}(\lambda)$ is modeled as by Bricaud et al. [1998], according to an empirical nonlinear relationship with respect to $\mathrm{Chl}$, which is written

$$
a_{\phi}(\lambda)=A_{\phi}(\lambda)(C h l)^{B(\lambda)} .
$$

With $B(\lambda)$ exponents all below 1 , the absorption coefficients per unit of chlorophyll are decreasing when the chlorophyll concentration within the water body is increasing. Then, the power emitted via fluorescence, $\Phi_{f}$, is simply obtained through

$$
\Phi_{f}=\phi_{f} \Phi_{a}
$$




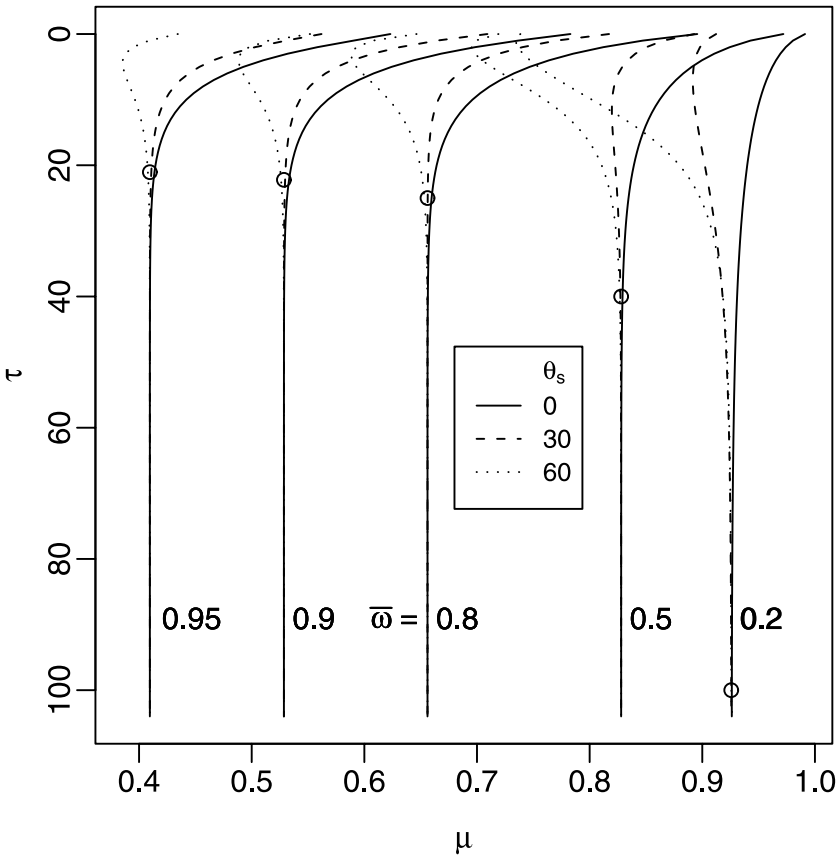

Figure C1. Evolution along with the optical depth $(\tau)$ of the average cosine $(\bar{\mu})$, for five water bodies characterized by their single albedo $(\varpi)$. Each group of curves corresponds to three solar angles. The open circles corresponds to a constant value (20) given to the scattering optical depth $\left(\tau_{b}\right)$.

where $\phi_{f}$ in this instance is given the value $0.6 \%$ (typical for near surface layer). The corresponding emitted photons are spread over the Gaussian band (half band width $18 \mathrm{~nm}$ ), and their number divided by 4 (because half of the emission is in the upward direction, and the average cosine for this isotropic radiance field is 0.5 ), and corrected according to
Maritorena et al. [2000, equation (7)] to obtain the upward plane irradiance due to fluorescence emitted by the upper layers which contribute to the signal. This irradiance is divided by $E_{d}\left(0^{-}, \lambda\right)$, the downward irradiance below the surface, to derive the contribution of the fluorescence signal which is to be added to the reflectance.

\section{Appendix C: Rate of Approach to the Asymptotic Regime}

[79] Instead of separately examining the evolutions with depth of each of the AOPs to detect the approach of the asymptotic limits (which nevertheless has been partly done), a global treatment amenable to generalization and understanding is more appropriate. This treatment is developed only for the elastic scattering domain. With this aim, specific RT computations have been effected by using a unique phase function (the mean particle phase function as computed by Mobley [1994] from Petzold's measurement), with $\varpi$ given the values $0.2,0.5,0.8,0.9$, and 0.95 , and with Sun-zenith angles $\theta_{s}=0^{\circ}, 30^{\circ}$, and $60^{\circ}$ (in a black sky). Fixing the wavelength or the chlorophyll concentration is no longer needed in this exercise.

[80] The asymptotic solution of the RTE has also been separately computed according to the numerical technique described by Prieur and Morel [1971] by using the same phase function and the same $\varpi$ values; it provides in each case the asymptotic and unique diffuse attenuation coefficient, $K_{\infty}$, (actually, the ratio $K_{\infty} / c$ as a function of $\varpi$ ), as well as other asymptotic values, such as the average cosine $\bar{\mu}_{\infty}$, through the exact relationship [see, e.g., Mobley, 1994]

$$
\bar{\mu}_{\infty}=(1-\varpi) /\left(K_{\infty} / c\right) .
$$

[81] Figure $\mathrm{C} 1$ shows as a function of the optical depth $\tau$, the evolution of the average cosine, $\mu$, taken as an example of AOP. The convergence of the three curves (each of them

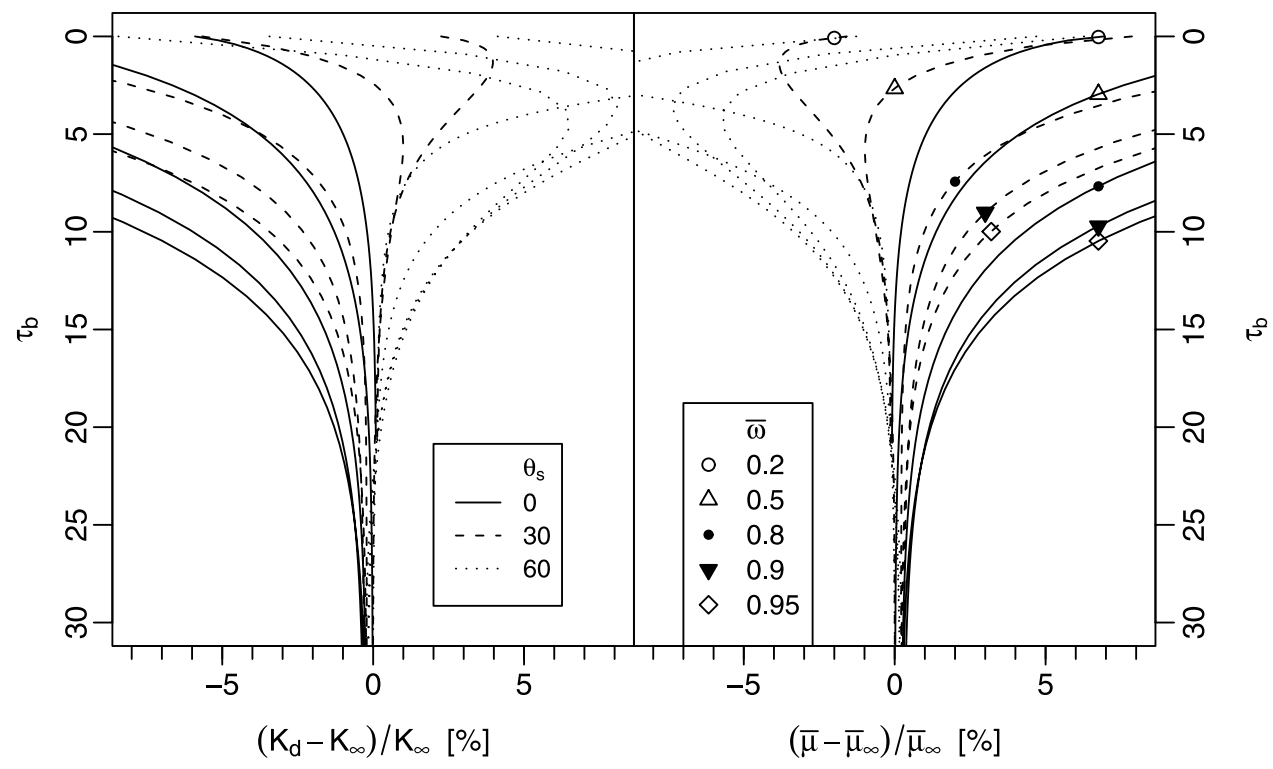

Figure C2. Relative differences between actual $K_{d}$ and $\bar{\mu}$ values and their asymptotic values as a function of the scattering optical depth $\left(\tau_{b}\right)$ for various water bodies (various $\varpi$ values) and three Sunzenith angles, as indicated. 
corresponding to the three initial $\theta_{s}$ values) within each group (corresponding to the various $\varpi$ values) does not occur at the same optical depth; clearly the localization of the convergence depends on the $\varpi$ values [see also Berwald et al., 1995, Figure 2; Piening and McCormick, 2003]. Other AOPs behave exactly the same with respect to the converging trends. Nevertheless, the zone of convergence seems to occur at the same $\tau_{b}$ level (open symbols in Figure C1). The rate of approach to the asymptotic state seems to be essentially governed by the $\tau_{b}$ parameter. To confirm this point, the quantities $\left.\left(\bar{\mu}-\bar{\mu}_{\infty}\right) / \bar{\mu}_{\infty}\right)$ and $\left(K_{d}-\right.$ $\left.K_{\infty}\right) / K_{\infty}$ have been formed by using the $\bar{\mu}_{\infty}$ and $K_{\infty}$ values that were independently derived via the asymptotic solution. The results are displayed on Figure C2. The computations through Hydrolight and the asymptotic solution agree remarkably well within $0.25 \%$ in the worst case (when $\varpi=0.95$ ) and better for other $\varpi$ values. By analyzing these graphs, the level at which an AOP is within a given percentage of its asymptotic value can be predicted. It is worth noting that the behavior depicted by Figure $\mathrm{C} 2$, in particular the convergence position, remains the same whatever the considered AOP, and is independent from the initial conditions. These conditions, however, size the initial $\left(\mathrm{AOP}-\mathrm{AOP}_{\infty}\right.$ ) differences, and thus the slope of the needed changes to reach the asymptotic values [see Zaneveld, 1989].

[82] By summarizing the results of the analysis, a $1.3 \%$ maximal difference with respect to the asymptotic value occurs at $\tau_{b}=20$, and $0.4 \%$ difference at $\tau_{b}=30$. By comparing these figures with the typical $\tau_{b}$ values reached in $Z_{\text {eu }}$ (Figure A2), the previous conclusions regarding the approach of the asymptotic regime can be simply interpreted. When $\mathrm{Chl}$ exceeds $2.5 \mathrm{mg} \mathrm{m}^{-3}$, the asymptotic regime is practically attained (within 1\%) everywhere in the spectrum at the $Z_{\text {eu }}$ level; for concentration below $1 \mathrm{mg} \mathrm{m}^{-3}$, the quasi-asymptotic regime does not appear before $2 Z_{\text {eu }}$ for the blue and green radiation, and not yet for the red radiations (but, as seen before, the Raman radiation actually has replaced the elastically transmitted radiation at this depth). For ultraoligotrophic waters $(\mathrm{Chl}=$ $0.03 \mathrm{mg} \mathrm{m}^{-3}$ ), this regime is established at a depth 3 or 4 times that of $Z_{\mathrm{eu}}$. The "pedagogically" [Gordon et al., 1993] interesting concept of asymptotic regime can conceal virtual or real situations, as already noted by Mobley [1994, p. 465], and exemplified by the present computation. Indeed, when $\varpi=0.9$ (or 0.8 ), and by using the asymptoticity criterion $\tau_{b}=20$, the associated $\tau_{a}$ values are 2.22 (or 5), which means that about $10 \%$ (or $0.67 \%$ ) of the initial photons remain not absorbed, and thus are still present to build the asymptotic light field. In contrast, with $\varpi=0.2$, and $\tau_{b}=20, \tau_{a}$ is as high as 80 , which means that only $10^{-35}$ photons have survived, so that the asymptotic regime in absorbing waters is more an elusive mathematical entity than a reality.

[83] Acknowledgments. We are grateful for the useful comments and suggestions from the two anonymous reviewers, and we are also in debt to Annick Bricaud for her scrutiny when examining the first draft of this paper and for her helpful comments.

\section{References}

Baker, K. S., and R. C. Smith (1982), Bio-optical classification and model of natural waters, Limnol. Oceanogr., 27, 500-509.
Bartlett, J. S., K. J. Voss, S. Sathyendranath, and A. Vodacek (1998), Raman scattering by pure water and seawater, Appl. Opt., 37, 3324-3332. Berwald, J., D. Stramski, C. D. Mobley, and D. A. Kiefer (1995), Influences of absorption and scattering on vertical changes in the average cosine of the underwater light field, Limnol. Oceanogr., 40, 1347-1357. Bricaud, A., and A. Morel (1987), Atmospheric corrections and interpretation of marine radiances in CZCS imagery: Use of a reflectance model, Oceanol. Acta, SP, 33-50.

Bricaud, A., A. Morel, M. Babin, K. Allali, and H. Claustre (1998), Variations of light absorption by suspended particles with chlorophyll a concentration in oceanic (case 1) waters: Analysis and implications for bio-optical models, J. Geophys. Res., 103, 31,033-31,044.

Bricaud, A., E. Bosc, and D. Antoine (2002), Algal biomass and sea surface temperature in the Mediterranean basin: Intercomparison of data from various satellite sensors, and implications for primary production estimates, Remote Sens. Environ., 81, 163-178.

Clark, D. K. (1981), Phytoplankton algorithms for the Nimbus-7 CZCS, in Oceanography From Space, edited by J. F. R. Gower, pp. 227-238, Plenum, New York.

Clarke, G. L., and G. C. Ewing (1974), Remote spectroscopy of the sea for biological production studies, in Optical Aspects of Oceanography, edited by N. G. Jerlov and E. Steemann-Nielsen, pp. 389-413, Academic, San Diego, Calif.

Cox, C., and W. Munk (1955), Some problems in optical oceanography, J. Mar. Res., 14, 63-78.

D’Ortenzio, F., S. Marullo, M. Ragni, M. Ribera d'Alcala, and R. Santoleri (2002), Validation of empirical SeaWiFS algorithms for chlorophyll-a retrieval in the Mediterranean Sea: A case study for oligotrophic seas, Remote Sens. Environ., 82, 79-94.

Gordon, H. R. (1989), Can the Lambert-Beer law be applied to the diffuse attenuation coefficient of ocean water?, Limnol. Oceanogr., 34, 13891409.

Gordon, H. R., and A. Morel (1983), Remote Assessment of Ocean Color for Interpretation of Satellite Visible Imagery: A Review, 114 pp., SpringerVerlag, New York.

Gordon, H. R., O. B. Brown, and M. M. Jacobs (1975), Computed relations between inherent and apparent optical properties of a flat homogeneous ocean, Appl. Opt., 14, 417-427.

Gordon, H. R., O. B. Brown, R. H. Ewans, J. W. Brown, R. C. Smith, K. S. Baker, and D. K. Clark (1988), A semi-analytic radiance model of ocean color, J. Geophys. Res., 93, 10,909-10,924.

Gordon, H. R., K. Ding, and W. Gong (1993), Radiative transfer in the ocean: Computations relating to the asymptotic and near-asymptotic daylight field, Appl. Opt., 32, 1606-1619.

Haltrin, V. H., and G. W. Kattawar (1991), Effects of Raman scattering and fluorescence on apparent optical properties of sea water, report, $72 \mathrm{pp}$., Texas A\&M Univ., College Station.

Kirk, J. T. O. (1984), Dependence of relationship between inherent and apparent optical properties of water on solar altitude, Limnol. Oceanogr., $29,350-356$.

Loisel, H., and A. Morel (1998), Light scattering and chlorophyll concentration in case 1 waters: A reexamination, Limnol. Oceanogr., 43, 847858 .

Maritorena, S., A. Morel, and B. Gentili (2000), Determination of the fluorescence quantum yield by oceanic phytoplankton in their natural habitat, Appl. Opt., 39, 6725-6737.

Mobley, C. D. (1994), Light and Water, 592 pp., Academic, San Diego, Calif.

Mobley, C. D., et al. (1993), Comparison of numerical models for computing underwater light fields, Appl. Opt., 32, 7484-7505.

Morel, A. (1974), Optical properties of pure water and pure seawater, in Optical Aspects of Oceanography, edited by N. G. Jerlov and E. Steemann-Nielsen, pp. 1-24, Academic, San Diego, Calif.

Morel, A. (1988), Optical modeling of the upper ocean in relation to its biogenous matter content (case 1 waters), J. Geophys. Res., 93, 10,74910,768 .

Morel, A. (1991), Light and photosynthesis: A spectral model with geochemical and climatological implications, Prog. Oceanogr., 26, $263-$ 306.

Morel, A., and D. Antoine (1994), Heating rate within the upper ocean in relation to its bio-optical state, J. Phys. Oceanogr., 24, 1652-1665.

Morel, A., and J.-F. Berthon (1989), Surface pigments, algal biomass profiles, and potential production of the euphotic layer: Relationships reinvestigated in view of remote sensing applications, Limnol. Oceanogr., $42,1545-1562$.

Morel, A., and B. Gentili (1996), Diffuse reflectance of oceanic waters, III, Implication of bidirectionality for the remote sensing problem, Appl. Opt., 35, 4850-4862.

Morel, A., and S. Maritorena (2001), Bio-optical properties of oceanic waters: A reappraisal, J. Geophys. Res., 106, 7163-7180. 
Morel, A., and J. L. Mueller (2002), Normalized water-leaving radiance and remote sensing reflectance: Bidirectional reflectance and other factors, in Ocean Optics Protocols for Satellite Ocean Color Sensor Validation, Rev. 3, vol. 2, edited by J. L. Mueller and G. S. Fargion, chap. 13, pp. $183-$ 210, NASA Goddard Space Flight Cent., Greenbelt, Md.

Morel, A., and L. Prieur (1977), Analysis of variations in ocean color, Limnol. Oceanogr., 22, 709-722.

Morel, A., and R. C. Smith (1974), Relation between total quanta and total energy for aquatic photosynthesis, Limnol. Oceanogr., 19, 591-600.

Morel, A., D. Antoine, and B. Gentili (2002), Bidirectional reflectance of oceanic waters: Accounting for Raman emission and varying particle scattering phase function, Appl. Opt., 41, 6289-6306.

Morrison, J. R. (2003), In situ determination of the quantum yield of phytoplankton chlorophyll a fluorescence: A simple algorithm, observations, and a model, Limnol. Oceanogr., 48, 618-631.

Neckel, H., and D. Labs (1984), The solar radiation between 3300 and 12500 A, Sol. Phys., 90, 205-258

O'Reilly, J. E., S. Maritorena, B. G. Mitchell, D. A. Siegel, K. L. Carder, S. A. Garver, M. Kahru, and C. McClain (1998), Ocean color chlorophyll algorithms for SeaWiFS, J. Geophys. Res., 103, 24,937-24,953.

Piening, B. D., and N. J. McCormick (2003), Asymptotic optical depths in source-free ocean waters, Appl. Opt., 42, 5382-5387.

Pope, R. M., and E. S. Fry (1997), Absorption spectrum (380-700 nm) of pure water, II, integrating cavity measurements, Appl. Opt., 36, 87108723 .
Preisendorfer, R. W. (1959), Theoretical proof of the existence of characteristic diffuse light in natural waters, J. Mar. Res., 18, 1-9.

Preisendorfer, R. W. (1961), Application of radiative transfer theory to light measurement in the sea, IUGG Monogr., 10, 11-30.

Prieur, L., and A. Morel (1971), Etude theorique du régime asymptotique: Relations entre caractéristiques optiques et coefficient d'extinction relatif à la pénétration de la lumière du jour, Cah. Oceanogr., 23, 35-47.

Ryther, J. H. (1956), Photosynthesis in the ocean as a function of light intensity, Limnol. Oceanogr., 1, 61-70.

Sathyendranath, S., and T. Platt (1997), Analytic model of ocean color, Appl. Opt., 36, 2620-2629.

Stramski, D., A. Bricaud, and A. Morel (2001), Modeling the inherent optical properties of the ocean based on the detailed composition of the planktonic community, Appl. Opt., 40, 2929-2945.

Waters, K. J. (1995), Effects of Raman scattering on the water-leaving irradiance, J. Geophys. Res., 100, 13,151-13,161.

Zaneveld, J. R. V. (1989), An asymptotic closure theory for irradiance in the sea and its inversion to obtain the inherent optical properties, Limnol. Oceanogr., 34, 1442-1452.

B. Gentili and A. Morel, Laboratoire d'Océanographie de Villefranche, Université Pierre et Marie Curie/CNRS, F-06238 Villefranche-sur-mer cedex, France. (bernard.gentili@obs-vlfr.fr; morel@obs-vlfr.fr) 